\title{
One-loop monodromy relations on single cuts
}

\author{
Alexander Ochirov, ${ }^{a}$ Piotr Tourkine ${ }^{b}$ and Pierre Vanhove ${ }^{c}$ \\ ${ }^{a}$ Higgs Centre for Theoretical Physics, School of Physics and Astronomy, \\ The University of Edinburgh, Peter Guthrie Tait Road, Edinburgh EH9 3FD, Scotland, U.K. \\ ${ }^{b}$ Department of Applied Mathematics and Theoretical Physics (DAMTP), \\ University of Cambridge, Wilberforce Road, Cambridge CB3 0WA, U.K. \\ ${ }^{c}$ Institut de Physique Théorique, \\ Université Paris Saclay, CNRS, F-91191 Gif-sur-Yvette, France \\ E-mail: alexander.ochirov@ed.ac.uk, pt373@cam.ac.uk, \\ pierre.vanhove@ipht.fr
}

ABSTRACT: The discovery of colour-kinematic duality has led to significant progress in the computation of scattering amplitudes in quantum field theories. At tree level, the origin of the duality can be traced back to the monodromies of open-string amplitudes. This construction has recently been extended to all loop orders. In the present paper, we dissect some consequences of these new monodromy relations at one loop. We use single cuts in order to relate them to the tree-level relations. We show that there are new classes of kinematically independent single-cut amplitudes. Then we turn to the Feynman diagrammatics of the string-theory monodromy relations. We revisit the string-theoretic derivation and argue that some terms, that vanish upon integration in string and field theory, provide a characterisation of momentum-shifting ambiguities in these representations. We observe that colour-dual representations are compatible with this analysis.

Keywords: Scattering Amplitudes, Superstrings and Heterotic Strings

ARXiv EPrint: 1707.05775 


\section{Contents}

1 Introduction 2

2 Single cut of one-loop amplitudes in field and string theory 3

2.1 Single cut and forward tree amplitudes 3

2.2 Single cut of one-loop string amplitudes 5

3 Momentum kernel in the forward limit $\quad 7$

3.1 Momentum kernel 7

3.2 Forward limit of the fundamental monodromy relation 9

$\begin{array}{lll}3.3 & \text { Fundamental monodromy relations at one loop } & 10\end{array}$

3.4 Forward limit of the momentum kernel 11

3.5 Monodromy relations and one-loop coefficients 13

4 Field-theory monodromy relations from string theory $\quad 14$

4.1 Loop integrand monodromy versus forward-amplitude monodromies 14

$\begin{array}{lll}\text { 4.1.1 String-theoretic boundary terms } & 15\end{array}$

4.1.2 Recovering the single-cut monodromy relation 16

$\begin{array}{lll}4.2 & \text { Dissecting the field theory limit of the string theory monodromies } & 17\end{array}$

$\begin{array}{lll}\text { 4.2.1 Role of boundary terms } & 17\end{array}$

$\begin{array}{lll}\text { 4.2.2 Planar versus non-planar refinement } & 18\end{array}$

$\begin{array}{lll}4.3 & \text { BCJ-compatibility of the string theory monodromies } & 19\end{array}$

$\begin{array}{lll}4.3 .1 & \text { Four-point case } & 20\end{array}$

$\begin{array}{lll}4.3 .2 & \text { Five-point case } & 21\end{array}$

5 Discussion $\quad 22$

$\begin{array}{ll}\text { A Forward limit parametrisation } & 23\end{array}$

B One-loop monodromies in open string $\quad 24$

B.1 Prescription for monodromies and complex logarithm 24

B.2 String theory one-loop monodromies at order $\alpha^{\prime} \quad 24$

$\begin{array}{lll}\text { B.2.1 Planar four-point relation } & 25\end{array}$

$\begin{array}{lll}\text { B.2.2 Non-planar four-point relation } & 27\end{array}$ 


\section{Introduction}

It is truly surprising that, more than sixty years after the basic principles of quantum field theory have been spelled out, we continue to discover profound facts about its perturbative expansion. The discovery of colour-kinematic duality $[1,2]$, for instance, provided a new way to organise the perturbation theory, which reveals a network of relationships between a variety of gauge, gravity and scalar field theories.

As a consequence of this duality, the number of independent tree-level colour-ordered amplitudes for $n$ gluons turned out to be $(n-3)$ !, whereas colour structure alone gives $(n-2)$ ! [3]. This aspect of the duality is beautifully explained via string theory by the monodromy properties of open-string amplitudes [4-6] and also in a Feynman-diagram context [7]. The duality holds in various settings [8-16] and up to the fourth loop order $[17,18]$. However, its extension to all orders [2] remains conjectural, and a modified implementation including contact terms has been proposed [19] to tackle the five-loop question, similar in spirit to the construction of [5].

In [20] two of the present authors have generalised the open-string monodromy relations to the multi-loop case and proposed corresponding explicit formulae. That construction generalised some relations for one-loop integrands made earlier in [21-25]. Implications for the colour-kinematic duality itself were left open: the purpose of this paper is to study some of these at the one-loop level.

We start by formulating in section 2 a different formulation of the monodromy relations, based on single cuts of field and string theory amplitudes. This leads us to some relations between regularised forward tree-amplitudes, somehow similar to these of [26, 27] based on the Cachazo, He and Yuan formalism (CHY) [28, 29]. These relations involve the forward limit of the so-called momentum kernel [30]. The equivalence between this formulation and the one of [20] is shown later on in section 4. Our main results are the following:

- We compute the co-rank of the momentum kernel in the forward limit: it increases from $(n-1)$ ! to $(n-1) !+(n-2)$ ! $+2(n-3)$ ! independent regulated $(n+2)$-point forward tree amplitudes. We identify three classes of independent tree amplitudes in the forward limit. These classes play a role in amplitudes where non-planar cuts are needed.

- We derive the forward monodromy relations from the monodromies in string theory. We re-interpret these monodromies, which actually produce exact integrand relations in the field-theory limit, by providing a precise form for the ambiguities that vanish upon integration in field theory. We explain the connection between such relations and the Bern-Carrasco-Johansson (BCJ) identities for forward tree amplitudes.

- We further show on a few examples how these integrand relations are always satisfied if a colour-dual representation ${ }^{1}$ of the loop amplitude exists. This is bootstrapped

\footnotetext{
${ }^{1}$ Colour-kinematic duality $[1,2]$ requires that the kinematic numerators of a gauge-theory amplitude satisfy (or can be massaged to satisfy) the same algebraic relations as their corresponding colour factors, for example, kinematic Jacobi identities. The duality-satisfying amplitude representation is then called colour-dual.
} 
up to a consistency constraint on the form a string-theoretic integrand that would produce colour-dual kinematic numerators. We observe the natural appearance of higher-order Jacobi-type relations.

We also present two appendices. In appendix A we give details on the way to perform the forward limit. In appendix B, we check that the monodromy relations hold to the first order in $\alpha^{\prime}$ for open-string at one loop. At the same time we solve the apparent discrepancy between the results of [20] and [31] by showing that it amounts to the different definitions of the branch cuts of the complex logarithms. Once this is taken into account, agreement is obtained.

\section{Single cut of one-loop amplitudes in field and string theory}

\subsection{Single cut and forward tree amplitudes}

In this section we discuss the reconstruction of one-loop amplitudes from single cuts. In the process we set up our conventions for the rest of the paper.

In any local unitary quantum field theory, for any number of spacetime dimensions, the Feynman Tree theorem [32,33] expresses one-loop amplitudes as integrals of tree-level amplitudes. The theorem prescribes to sum over all possible ways of cutting an internal propagator. Its application to construct one-loop amplitudes [34] can be aided by using MHV vertices [35]. In [36] it was further shown that a one-loop amplitude can be obtained by integration of the single-cut amplitude over a special contour. This amounts to a change of the $i \varepsilon$-prescription in the propagator, which is equivalent to the Feynman Tree theorem and is the basis of the "loop-tree" duality method [36-40].

Moreover, in [41-43] it was shown how a QCD amplitude can be calculated by taking single cuts in $D=4-2 \epsilon$. We follow the logic of these works for relating one-loop amplitudes and their single cuts.

Consider a colour-stripped $n$-point amplitude in $D$ dimensions,

$$
A_{n}^{(1)}\left(p_{1}, \ldots, p_{n}\right)=\int d^{D} \ell \frac{\mathcal{N}(\ell)}{\prod_{i=0}^{n-1} d_{i}}, \quad d_{i}=\left(\ell+p_{1 \ldots i}\right)^{2}-m_{i}^{2}+i \varepsilon,
$$

with $\varepsilon>0, p_{1 \ldots k}:=p_{1}+\cdots+p_{k}$, and the momentum conservation condition is $p_{1 \ldots n}=0$. The loop measure is defined by

$$
\int \pi^{D} \ell:=\int \frac{d^{D} \ell}{(2 \pi)^{D}}
$$

The single-cut operator $\delta_{d_{i}}^{P}$ acts by replacing, in the integrand of the one-loop integral, the propagator $d_{i}$ between the legs $p_{i}$ and $p_{i+1}$ by the operator $2 \pi \delta^{(+)}\left(\left(\ell+p_{1 \ldots i}\right)^{2}-m_{i}^{2}\right)=$ $2 \pi \delta\left(\left(\ell+p_{1 \ldots i}\right)^{2}-m_{i}^{2}\right) \theta\left(\left(\ell+p_{1 \ldots i}\right)^{0}\right)$, so that

$$
\delta_{d_{i}}^{P} A_{n}^{(1)}\left(p_{1}, \ldots, p_{n}\right):=\int \pi^{D} \ell 2 \pi \delta^{(+)}\left(d_{i}\right) A_{n+2}^{1-\operatorname{cut}}\left(\ell+p_{1 \ldots i}, p_{i+1}, \ldots, p_{n+i},-\left(\ell+p_{1 \ldots i}\right)\right),
$$

where the single-cut amplitude reads

$$
A_{n+2}^{1-\mathrm{cut}}\left(\ell+p_{1 \ldots i}, p_{i+1}, \ldots, p_{n+i},-\left(\ell+p_{1 \ldots i}\right)\right)=\frac{\mathcal{N}(\ell)}{d_{0} d_{1} \cdots d_{i-1} d_{i+1} \cdots d_{n-1}} .
$$


By shifting the loop momentum we rewrite the cut propagator as $d_{i}=\ell^{2}-m_{i}^{2}$ and the single-cut operator of one-loop integral reads

$$
\delta_{d_{i}}^{P} A_{n}^{(1)}\left(p_{1}, \ldots, p_{n}\right)=\int d^{D} \ell 2 \pi \delta^{(+)}\left(\ell^{2}-m_{\rho(i)}^{2}\right) A_{n+2}^{1-\mathrm{cut}}\left(\ell, p_{i+1}, \ldots, p_{n+i},-\ell\right),
$$

where the external-particle labels are understood modulo $n$.

The one-loop amplitude is also expressible in terms of irreducible numerators [44]:

$$
A_{n}^{(1)}\left(p_{1}, \ldots, p_{n}\right)=\sum_{r} \sum_{i_{1}<\cdots<i_{r}} \int d^{D} \ell \frac{\Delta_{i_{1}, \ldots, i_{r}}(\ell)}{d_{i_{1}} \ldots d_{i_{r}}}
$$

where the index $r$ runs over all possible irreducible topologies. For instance, in $D=4-2 \epsilon$ we have $r=5$ for pentagons, $r=4$ for boxes, $r=3$ for triangles, $r=2$ for bubbles and $r=1$ for tapdole integral functions [45, 46]. Each irreducible numerator $\Delta_{i_{1}, \ldots, i_{r}}$ is obtained by computing residues on the locus of vanishing of its corresponding $r$ propagators, $d_{i_{1}}=\cdots=d_{i_{r}}=0[47,48]$. There are up to $\frac{n !}{r !(n-r) !}$ massive $r$-gon integral functions in an $n$-point amplitude.

The single cut of a one-loop amplitude with propagator $d_{i}$ removed then decomposes as

$$
A_{n+2}^{1 \text {-cut }}\left(\ell+p_{1 \ldots i}, p_{i+1}, \ldots, p_{n+i},-\left(\ell+p_{1 \ldots i}\right)\right)=\sum_{r} \sum_{i<i_{1}<\cdots<i_{r-1}} \frac{\Delta_{i, i_{1}, \ldots, i_{r-1}}(\ell)}{d_{i_{1}} \ldots d_{i_{r-1}}} .
$$

In other words, only the master integrals that have a propagator between the legs $p_{i}$ and $p_{i+1}$ contribute to the single cut. Such a single-cut amplitude can be further cut to gradually obtain all its irreducible numerators. In this sense the knowledge of the single cuts of a one-loop amplitude allows to reconstruct it in full [41-43].

It is tempting to identify a single cut $A_{n+2}^{1 \text {-cut }}\left(\ell, p_{1}, \ldots, p_{n},-\ell\right)$ with the colour-stripped tree-level amplitude $A_{n+2}^{\text {tree }}\left(\ell, p_{1}, \ldots, p_{n},-\ell\right)$ in the forward limit, summed over the particle crossing the cut. However, such a forward limit is divergent. Indeed, if we choose to parametrise the $(n+2)$ on-shell momenta with a complex parameter $z$ such that the last two momenta

$$
q_{2}=p_{n+1}(z) \underset{z \rightarrow 0}{\longrightarrow}-\ell, \quad q_{1}=p_{n+2}(z) \underset{z \rightarrow 0}{\longrightarrow} \ell
$$

depend linearly on $z$, then there are two types of singular contributions:

1. single cuts of bubbles on external legs contain $1 /\left(q_{1}+q_{2}+p_{i}\right)^{2} \underset{z \rightarrow 0}{\longrightarrow} \mathcal{O}(1 / z)$,

2. single cuts of massless tadpoles, depending on the propagator structure, such diagrams diverge as $1 /\left(q_{1}+q_{2}\right)^{2} \underset{z \rightarrow 0}{\longrightarrow} \mathcal{O}\left(1 / z^{2}\right)$, or even as $1 /\left(\left(q_{1}+q_{2}\right)^{2}\left(q_{1}+q_{2}+p_{i}\right)^{2}\right) \underset{z \rightarrow 0}{\longrightarrow}$ $\mathcal{O}\left(1 / z^{3}\right)$

The corresponding diagrams are depicted in figure 1 (also see e.g. [49, §7.1]). Neither of these divergences can arise from the single cut, because they correspond to cuts of the propagator renormalisation or wave-function renormalisation of external legs. Such divergences cancel in supersymmetric gauge theories [34, 50, 51]. Nevertheless, they are present in forward tree amplitudes simply at the level of Feynman graphs. 


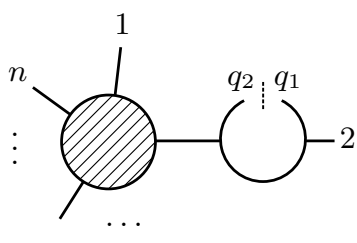

$1 / z$

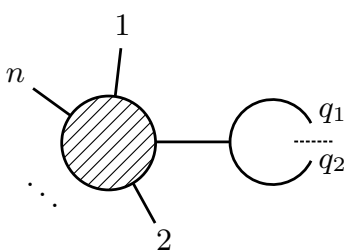

$1 / z^{2}$

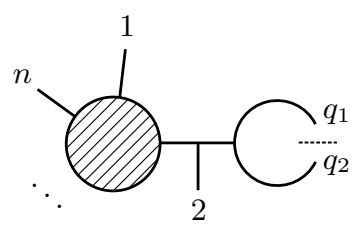

$1 / z^{3}$

Figure 1. Wave-function renormalisation and tadpole graphs divergent in the forward limit.

One could in principle remove such diagrams [52, 53], at the expense of losing gauge invariance. Another way is to regularise the forward limit [43] and then extract the regular part of $(n+2)$-point tree-level amplitude:

$$
A_{n+2}^{\text {tree }}\left(\ell, p_{1}, \ldots, p_{n},-\ell+z \hat{q}\right)=\frac{\alpha_{3}}{z^{3}}+\frac{\alpha_{2}}{z^{2}}+\frac{\alpha_{1}}{z}+\hat{A}_{n+2}^{\text {tree }}\left(\ell, p_{1}, \ldots, p_{n},-\ell\right)+\mathcal{O}(z) .
$$

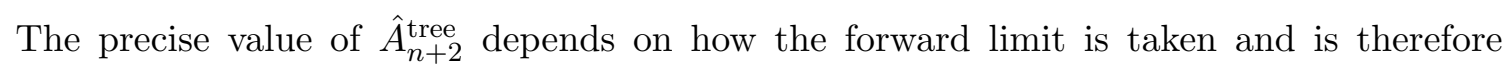
scheme-dependent. The forward-limit parametrisation that we use in the massless case is detailed in appendix A. However, it is clear that if we compute any unitarity cut corresponding to a non-zero topology by cutting any further propagators, then the divergent contributions and any scheme dependence will be projected out. In other words, such contributions have zero physical cuts. Therefore, we are allowed to identify the regularised finite part of the forward tree amplitudes with the single cut of the propagator $d_{0}=\ell^{2}+i \varepsilon=0$. We thus have a decomposition for the state-summed forward tree

$$
\sum_{h}(-1)^{2 h} \hat{A}_{n+2}^{\text {tree }}\left(\ell^{h}, p_{1}, \ldots, p_{n},-\ell^{\bar{h}}\right)=\sum_{r} \sum_{i_{1}<\cdots<i_{r-1}} \frac{\Delta_{0, i_{1}, \ldots, i_{r-1}}(\ell)}{d_{i_{1}} \ldots d_{i_{r-1}}} .
$$

Taking residues of the forward tree amplitude with respect to the loci $d_{i_{1}}=d_{i_{2}}=d_{i_{3}}=0$ then gives the numerators $\Delta_{0, i_{1}, \ldots, i_{3}}(\ell)$, in the same way as the conventional quadruple cut [47] defined by $d_{0}=d_{i_{1}}=d_{i_{2}}=d_{i_{3}}=0$ of the one-loop amplitude. Similarly, residues with respect to the loci $d_{i_{1}}=d_{i_{2}}=0$ match the triple cuts defined by $d_{0}=d_{i_{1}}=d_{i_{2}}=0$, and so on. All the information about the irreducible numerator of the one-loop amplitude is contained in its (regularised) forward tree amplitudes.

The forward tree amplitude $\hat{A}_{n+2}^{\text {tree }}$ in eq. (2.10) lacks contributions from the one-loop integral functions that do not contain the propagator $d_{0}$ between $p_{1}$ and $p_{n}$. There are hence only $\frac{(n-1) !}{r !(n-r-1) !}$ irreducible numerators for $r$-gons in a given ordered forward amplitude. Of course, the full set of one-loop numerators (and the integral coefficients therein) is recovered by considering all the external-leg permutations of forward tree amplitudes.

\subsection{Single cut of one-loop string amplitudes}

Cutkosky rules in superstring field theory have been recently revisited in $[54,55]$, but not from a worldsheet perspective. In this section we aim at doing so, as it will be needed later in section 4.

Intuitively, it is clear a single cut of a one-loop string theory diagram should produce a diagram with tree-level topology and two additional states. So we start by inserting a 
cutting operator in a one-loop open-string amplitude of the form $\left(\ell^{2}-m^{2}\right) \delta\left(\ell^{2}-m^{2}\right) \Theta\left(\ell^{0}\right)$. We will then prove how this forces the integration of the moduli of the annulus to localise onto the pinched annulus, which is a disk with two points identified. In the ambitwistor formulation [56-58] of the CHY formula [28, 29], this localisation is automatically implemented by the loop-level scattering equations [59-61]. For us here, the process is different - we really look specifically at the cut of a string amplitude.

We start with a generic bosonic open-string amplitude in the representation where the loop momentum is not integrated [62] (the notation and conventions follow [20])

$$
\begin{aligned}
& \mathcal{A}(\alpha \mid \beta)=\int_{0}^{\infty} d t \int d^{D} \ell \int_{\Delta_{\alpha \mid \beta}} d^{n-1} \nu e^{-\pi \alpha^{\prime} t \ell^{2}-2 i \pi \alpha^{\prime} \ell \cdot \sum_{i=1}^{n} p_{i} \nu_{i}} \\
& \times \prod_{1 \leq r<s \leq n} f\left(e^{-2 \pi t}, \nu_{r}-\nu_{s}\right) \times e^{-\alpha^{\prime} p_{r} \cdot p_{s} G\left(\nu_{r}-\nu_{s}\right)},
\end{aligned}
$$

where the domain of integration $\Delta_{\alpha \mid \beta}$ is the union of the domains of integration specified by $0 \leq \Im \mathrm{m}\left(\nu_{\alpha(1)}\right) \leq \cdots \leq \Im \mathrm{m}\left(\nu_{\alpha(p)}\right)=t$ for $\Re \mathrm{e}\left(\nu_{i}\right)=0$ and $t \geq \Im \mathrm{m}\left(\nu_{\beta(p+1)}\right) \geq \cdots \geq$ $\Im \mathrm{m}\left(\nu_{\beta(n)}\right) \geq 0$ for $\Re \mathrm{e}\left(\nu_{i}\right)=\frac{1}{2}$. The non-zero mode part of the Green's function is given by

$$
G(\nu)=-\log \frac{\vartheta_{1}(\nu \mid i t)}{\vartheta_{1}^{\prime}(0)}=-\log \frac{\sin (\pi \nu)}{\pi}-4 \sum_{n \geq 1} \frac{q^{n}}{1-q^{n}} \frac{\sin ^{2}(n \pi \nu)}{n},
$$

where we have set $q=\exp (-2 \pi t)$. Note that since we have introduced the loop momentum, there is no zero-mode contribution to this Green's function.

Now we consider the insertion of the single-cut operator $\alpha^{\prime} \ell^{2} \delta\left(\alpha^{\prime} \ell^{2}\right) \Theta\left(\ell^{0}\right):=$ $\alpha^{\prime} \ell^{2} \delta^{(+)}\left(\alpha^{\prime} \ell^{2}\right)$ in the integrand, and we shall show that the integration localises at the pinched surface, which is given by the infinitely long annulus for which $t=\infty$. In the one-loop amplitude with the insertion of the single-cut operator,

$$
\begin{aligned}
\mathcal{A}(\alpha \mid \beta)^{\mathrm{proj}}:=\int d^{D} \ell \int_{0}^{\infty} d t \int_{\Delta_{\alpha \mid \beta}} d^{n-1} \nu e^{-\pi \alpha^{\prime} t \ell^{2}-2 i \pi \alpha^{\prime} \ell \cdot \sum_{i=1}^{n} p_{i} \nu_{i}} \times \alpha^{\prime} \ell^{2} \delta^{(+)}\left(\alpha^{\prime} \ell^{2}\right) \\
\times \prod_{1 \leq r<s \leq n} f\left(e^{-2 \pi t}, \nu_{r}-\nu_{s}\right) \times e^{-\alpha^{\prime} p_{r} \cdot p_{s} G\left(\nu_{r}-\nu_{s}\right)},
\end{aligned}
$$

the second line has a $q$-expansion due to the excited string modes propagating in the loop. Formally, integrating over the $\nu_{i}$ variables leads to an expression of the form

$$
\mathcal{A}(\alpha \mid \beta)^{\mathrm{proj}}=\int \pi^{D} \ell \int_{0}^{\infty} d t \alpha^{\prime} \ell^{2} \delta^{(+)}\left(\alpha^{\prime} \ell^{2}\right) \sum_{n \geq 0} c_{n}(t) e^{-\pi \alpha^{\prime} \ell^{2} t-2 \pi n t} .
$$

We can then reabsorb the loop-momentum dependence of the exponential by rescaling the proper time $t$ :

$$
\mathcal{A}(\alpha \mid \beta)^{\text {proj }}=\int_{0}^{\infty} d t \int d^{D} \ell \sum_{n \geq 0} \frac{\alpha^{\prime} \ell^{2} \delta^{(+)}\left(\alpha^{\prime} \ell^{2}\right)}{\alpha^{\prime} \ell^{2}-2 n} c_{n}\left(\frac{t}{\alpha^{\prime} \ell^{2}-2 n}\right) e^{-\pi t} .
$$

The delta-function insertion projects the integral on the $n=0$ sector,

$$
\mathcal{A}(\alpha \mid \beta)^{\text {proj }}=\int_{0}^{\infty} d t \int d^{D} \ell \delta^{(+)}\left(\alpha^{\prime} \ell^{2}\right) c_{0}\left(\frac{\alpha^{\prime} t}{\alpha^{\prime} \ell^{2}}\right) e^{-\pi t} .
$$


This final expression is identical to what would have happened if we had chosen the leading term $\mathcal{O}(1)$ term in the $q$-expansion. This is effectively equivalent to having set $q=0 \Leftrightarrow t=+\infty$ in the one-loop single-cut string integrand, which eventually proves our initial claim.

\section{Momentum kernel in the forward limit}

In this section we study the monodromy relations satisfied by the colour-stripped forward tree-level amplitudes in gauge theory.

\subsection{Momentum kernel}

Colour-stripped open-string disc amplitudes (denoted by a calligraphic $\mathcal{A}$ ) satisfy the following fundamental monodromy relation [63] (with $p_{r \ldots s}=\sum_{i=r}^{s} p_{i}$ )

$$
\mathcal{A}_{n+2}^{\text {tree }}\left(p_{1}, p_{2}, \ldots, p_{n+2}\right)+\sum_{i=2}^{n+1} e^{i \alpha^{\prime} p_{1} \cdot p_{2 \cdots i}} \mathcal{A}_{n+2}^{\text {tree }}(p_{2}, \ldots, \underbrace{p_{1}}_{\text {position } i}, \ldots, p_{n+2})=0 .
$$

Such relations, obtained by circulating a single momentum $p_{i}$, generate all the monodromy relations between the open string amplitudes [4-6]. The external states can be massive or massless, because, as explained in [64], monodromy relations between tree amplitudes are generic properties common to any tree amplitude independently of the details of the theory.

From now we set $q_{1}=p_{n+2}$ and $q_{2}=p_{n+1}$. These momenta can be massless or massive and satisfy the momentum conservation relation

$$
p_{1}+\cdots+p_{n}=-q_{1}-q_{2} .
$$

The amplitudes being real, taking the real and imaginary part of (3.1) simply amounts to taking cosines or sines from the phases. In the limit of infinite string tension, $\alpha^{\prime} \rightarrow 0$, one obtains relations between colour-ordered field theory amplitude $A\left(q_{1}, p_{1}, \ldots, p_{n}, q_{2}\right)$ and its external-leg permutations.

The leading order- $\alpha^{\prime}$ contribution of the real part of (3.1), using that $\cos \left(\alpha^{\prime} p \cdot q\right) \simeq 1$, leads to the photon-decoupling identities

$$
\begin{gathered}
A_{n+2}^{\text {tree }}\left(q_{1}, p_{1}, p_{2}, \ldots, p_{n}, q_{2}\right)+\sum_{i=2}^{n+1} A_{n+2}^{\text {tree }}(p_{2}, \ldots, \underbrace{p_{1}}_{\text {position } i}, \ldots, p_{n}, q_{2}, q_{1})=0, \\
A_{n+2}^{\text {tree }}\left(q_{1}, p_{1}, p_{2}, \ldots, p_{n}, q_{2}\right)+\sum_{i=2}^{n+1} A_{n+2}^{\text {tree }}(p_{1}, \ldots, \underbrace{q_{1}}_{\text {position } i}, \ldots, p_{n}, q_{2})=0 .
\end{gathered}
$$

Furthermore, since $\sin \left(\alpha^{\prime} p \cdot q\right) \simeq \alpha^{\prime} p \cdot q$, the imaginary part of (3.1) at leading order in $\alpha^{\prime}$ leads to so-called fundamental BCJ relations [65] that imply the full range of the BCJ kinematic relations [1] between gauge-theory amplitudes. Circulating the momentum $p_{1}$

$$
\sum_{i=1}^{n} p_{1} \cdot\left(q_{1}+p_{2 \cdots i}\right) A_{n+2}^{\text {tree }}(p_{2}, \ldots, \underbrace{p_{1}}_{\text {position } i}, \ldots, p_{n}, q_{2}, q_{1})=0,
$$


or circulating $q_{1}$

$$
\sum_{i=1}^{n} q_{1} \cdot p_{1 \ldots i} A_{n+2}^{\text {tree }}(p_{1}, \ldots, \underbrace{q_{1}}_{\text {position }}, \ldots, p_{n}, q_{2})=0
$$

and circulating $q_{2}$

$$
q_{2} \cdot q_{1} A_{n+2}^{\text {tree }}\left(q_{1}, q_{2}, p_{1}, \ldots, p_{n}\right)+\sum_{i=1}^{n-1} q_{2} \cdot\left(q_{1}+p_{1 \ldots i}\right) A_{n+2}^{\text {tree }}(p_{1}, \ldots, \underbrace{q_{2}}_{\text {position } i+1}, \ldots, p_{n}, q_{1})=0 .
$$

These relations generate all the monodromy BCJ relations satisfied by the colour-ordered amplitudes. The resulting equations are concisely rewritten using the momentum-kernel formalism [30]. The power of this formalism is that in addition to the BCJ relations it provides simultaneous and gauge-invariant treatment of the KLT construction [66] for gravity amplitudes out of gauge-theory amplitudes. Because of the two marked momenta $q_{1}$ and $q_{2}$ we have three kinds of momentum kernels and corresponding kinematic relations.

- Monodromy relations acting only on an external momentum $p_{i}$ and neither $q_{1}$ nor $q_{2}$ :

$$
\sum_{\sigma \in \mathfrak{S}_{n}} \mathcal{S}[\sigma(1, \ldots, n) \mid \beta(1, \ldots, n)]_{q_{2}} A_{n+2}^{\text {tree }}\left(q_{1}, \sigma\left(p_{1}, \ldots, p_{n}\right), q_{2}\right)=0,
$$

where $\mathfrak{S}_{n}$ is the set of permutations of $n$ elements, $\beta$ is any permutation of legs $1, \ldots, n$, and the sum runs over all permutations $\sigma$ of these legs. The momentum kernel $\mathcal{S}$ is given by $[30,67,68]^{2}$

$$
\mathcal{S}[\sigma(1, \ldots, n) \mid \beta(1, \ldots, n)]_{q}:=\prod_{i=1}^{n}\left(q \cdot p_{\sigma(i)}+\Theta_{i}(\sigma, \beta)\right),
$$

where the quantity $\Theta_{i}(\sigma, \beta)$ is defined by

$$
\Theta_{i}(\sigma, \beta)=\sum_{j=i+1}^{n} \theta(\sigma(i), \beta(j)) p_{\sigma(i)} \cdot p_{\beta(j)} .
$$

Here $\theta(i, j)$ is 1 if the ordering of the legs $i$ and $j$ is opposite in the sets $\left\{i_{1}, \ldots, i_{k}\right\}$ and $\left\{j_{1}, \ldots, j_{k}\right\}$ and 0 if the ordering is the same. Moreover, the reference momentum $q$ should not belong to the set $\{1, \ldots, n\}$, hence $\Theta_{i}(\sigma, \beta)$ does not depend on $q_{1}$.

- The monodromy relations acting on one of the special momentum, say $q_{1}$,

$$
\sum_{\sigma \in \mathfrak{S}_{n}} \mathcal{S}\left[\sigma\left(q_{1}, 2, \ldots, n\right) \mid \beta\left(q_{1}, 2, \ldots, n\right)\right]_{q_{2}} A_{n+2}^{\text {tree }}\left(p_{1}, \sigma\left(q_{1}, p_{2}, \ldots, p_{n}\right), q_{2}\right)=0 .
$$

\footnotetext{
${ }^{2}$ There is some freedom in the expression for the momentum kernel due to the various different ways of organising the KLT relation between closed-string amplitudes and open-string amplitudes [66]. In this work we follow the contour deformation used in [30] which leads to flip in the ordering of the legs in the right-moving amplitude compared to the left moving amplitude as given in [30, eq. (2.16)]. A different ordering of the leg in the right-moving amplitude will result in a different form for the momentum kernel as used for example in $[29,69,70]$. These different forms are equivalent since they lead to the equivalent linear relations between the colour-ordered gauge theory amplitudes, and the same gravitational amplitudes.
} 
- The monodromy relations moving both special momenta $q_{1}$ and $q_{2}$

$$
\begin{aligned}
\sum_{\sigma \in \mathfrak{S}_{n}} \mathcal{S}\left[\sigma\left(q_{2}, q_{1}, 2, \ldots, n-1\right) \mid\right. & \left.\beta\left(q_{2}, q_{1}, 2, \ldots, n-1\right)\right]_{p_{1}} \\
\times & A_{n+2}^{\text {tree }}\left(p_{n}, \sigma\left(q_{2}, q_{1}, p_{2}, \ldots, p_{n}\right), p_{1}\right)=0 .
\end{aligned}
$$

\subsection{Forward limit of the fundamental monodromy relation}

Now we consider the forward limit of the monodromy relation (3.7). It is obtained by taking the limit $q_{1}+q_{2} \rightarrow 0$ so that $p_{1}+\cdots+p_{n} \rightarrow 0$. We set $q_{1} \rightarrow \ell$ and $q_{2} \rightarrow-\ell$. Care must be exercised when taking the forward limit as the tree amplitudes can develop divergences.

If the forward limit is parametrised by a parameter $z \rightarrow 0$, the discussion of section 2.1 implies that the tree amplitude develops at most third-order poles in $z$ :

$$
\lim _{z \rightarrow 0} A_{n+2}^{\text {tree }}\left(q_{1}, p_{1}, \ldots, p_{n}, q_{2}\right)=\frac{\alpha_{3}}{z^{3}}+\frac{\alpha_{2}}{z^{2}}+\frac{\alpha_{1}}{z}+\hat{A}_{n+2}^{\text {tree }}\left(\ell, p_{1}, \ldots, p_{n},-\ell\right)+\mathcal{O}(z) .
$$

The momentum kernel is a polynomial in the loop momentum. It does not have poles and has a $z$-expansion of at most second order in $z$ for fundamental monodromy relations, and at most of the order $z^{2 n}$ for the momentum kernel in eq. (3.8). The forward limit of the monodromy relation (3.7) reads

$$
\left(\mathcal{S}(\ell)+z \mathcal{S}^{(1)}(\ell)+z^{2} \mathcal{S}^{(2)}(\ell)+z^{3} \mathcal{S}^{(3)}(\ell)+\mathcal{O}\left(z^{4}\right)\right)\left(\frac{\alpha_{3}}{z^{3}}+\frac{\alpha_{2}}{z^{2}}+\frac{\alpha_{1}}{z}+\hat{A}_{n+2}^{\text {tree }}+\mathcal{O}(z)\right)=0,
$$

leading to the system of equations

$$
\begin{aligned}
\mathcal{S}(\ell) \alpha_{3} & =0, \\
\mathcal{S}(\ell) \alpha_{2}+\mathcal{S}^{(1)}(\ell) \alpha_{3} & =0, \\
\mathcal{S}(\ell) \alpha_{1}+\mathcal{S}^{(1)}(\ell) \alpha_{2}+\mathcal{S}^{(2)}(\ell) \alpha_{3} & =0, \\
\mathcal{S}(\ell) \hat{A}^{\text {tree }}+\mathcal{S}^{(1)}(\ell) \alpha_{1}+\mathcal{S}^{(2)}(\ell) \alpha_{2}+\mathcal{S}^{(3)}(\ell) \alpha_{3} & =0 .
\end{aligned}
$$

The last equation shows that the finite part of forward tree amplitude satisfies an inhomogeneous monodromy relation of the form

$$
\mathcal{S}(\ell) \hat{A}_{n+2}^{\text {tree }}=R_{n+2}(\ell) .
$$

where $R_{n+2}$ is defined in terms of the $\mathcal{S}^{(i)}$ and $\alpha_{i}$. For the case of the fundamental BCJ monodromy relation we have

$$
\sum_{i=1}^{n} p_{1} \cdot\left(\ell+p_{2 \ldots i}\right) \hat{A}_{n+2}^{\text {tree }}(p_{2}, \ldots, \underbrace{p_{1}}_{\text {position } i}, \ldots, p_{n},-\ell, \ell)=R_{n+2}\left(p_{1}, \ldots, p_{n}, \ell\right),
$$

with equivalent statements, mutatis mutandis, for the forward limits of (3.10) and (3.11).

Since the modified relation (3.16) depends on the regularisation used to extract the finite part, we need to analyse the effect of the regularisation on the monodromy relations. 
As explained in section 2.1, the residues of the regularised forward tree amplitudes give the same residues from the multiple cut of the one-loop amplitude. The right-hand side of the regularised monodromy relation (3.16) does not contribute the residues because the divergences arise only from the tadpole and wave-function renormalisation contributions in figure 1. Therefore the monodromy relations between the various residues and thus the irreducible numerators are independent of the regularisation and non-ambiguous.

It is interesting to note that there exists a regularisation for which $R_{n+2}\left(p_{1}, \ldots, p_{n}, \ell\right)=$ 0 . This is the one used for the partial amplitudes $a\left(p_{1}, \ldots, p_{n},-,+\right)$ in $[26,27,60]$ which are regulated using the $\mathcal{Q}$-cut prescription [71] or the CHY prescription $[60,72,73]$. It is shown in these works that the partial amplitudes satisfy the fundamental BCJ monodromy relations (3.4) with a vanishing right-hand side. Moreover, $\mathcal{N}=4$ super-Yang-Mills amplitudes are completely free of forward-limit singularities [34, 50, 51]. From now on we will assume that our forward tree amplitudes are regularised in the way that preserves the form of the fundamental BCJ relations.

\subsection{Fundamental monodromy relations at one loop}

Now we assume that the fundamental monodromy relations in eq. (3.4) (and its permutations) hold for the forward tree amplitudes:

$$
\begin{gathered}
\sum_{i=1}^{n} p_{1} \cdot\left(\ell+p_{2 \ldots i}\right) \hat{A}_{n+2}^{\text {tree }}(p_{2}, \ldots, \underbrace{p_{1}}_{\text {position } i}, \ldots, p_{n},-\ell, \ell)=0, \\
\sum_{i=1}^{n} \ell \cdot p_{1 \ldots i} \hat{A}_{n+2}^{\text {tree }}(p_{1}, \ldots, \underbrace{\ell}_{\text {position } i+1}, \ldots, p_{n},-\ell)=0, \\
\sum_{i=1}^{n-1} \ell \cdot\left(\ell+p_{1 \ldots i}\right) \hat{A}_{n+2}^{\text {tree }}(p_{1}, \ldots, \underbrace{-\ell}_{\text {position } i+1}, \ldots, p_{n}, \ell)=-\ell^{2} \hat{A}_{n+2}^{\text {tree }}\left(\ell,-\ell, p_{1}, \ldots, p_{n}\right) .
\end{gathered}
$$

Since at one loop there are only two special legs, with the incoming and outgoing loop momentum, one can find monodromy relations amongst single cuts of planar graphs only. The forward limits of the monodromy relations in eqs. (3.17b) and (3.17c) mix single cuts of planar and non-planar amplitudes. But since one can always express the field-theory non-planar amplitudes as a combination of planar amplitudes [74], we do not have to study these equations in too much detail. Of course, at higher loops the non-planar contributions cannot be avoided [20]. ${ }^{3}$

An explicit solution of the monodromy relations between tree amplitudes expressing the amplitudes $\hat{A}_{n+2}^{\text {tree }}\left(\ell, \alpha, p_{1}, \beta,-\ell\right)$ in the minimal basis of $(n-3)$ ! amplitudes with leg 1 next to $\ell, \hat{A}_{n+2}^{\text {tree }}\left(\ell, p_{1}, \gamma,-\ell\right)$, can be read off from [1, eq. $\left.(4.22)\right]$ :

$$
\hat{A}_{n+2}^{\text {tree }}\left(\ell, \alpha, p_{1}, \beta,-\ell\right)=\sum_{\sigma \in S(\alpha) \omega \beta} \hat{A}_{n+2}^{\text {tree }}\left(\ell, p_{1}, \sigma,-\ell\right) \prod_{i=1}^{|\alpha|} \frac{\mathcal{F}_{\ell}\left(\sigma, \alpha_{i}\right)}{s_{\ell \alpha_{1} \ldots \alpha_{i}}},
$$

\footnotetext{
${ }^{3}$ The integrand monodromy relations in string theory [20,31] relate planar and non-planar amplitudes by distributing the external legs on the various boundaries of the open-string amplitude. It should be noted that these relations do not change the master topology of the open-string vacuum graphs but just the position of the external legs inside a given topology.
} 
where $\alpha$ is a short-hand notation for the permutations of the external legs $p_{\alpha(2)}, \ldots, p_{\alpha(r)}$, $\beta$ for the permutations of the external legs $p_{\beta(r+1)}, \ldots, p_{\beta(n)}$. The sum is over the permutations $\gamma$ running over the shuffle products between $\beta$ and the permutations of $\alpha$. There are at most $(n-1)$ ! terms in the expansion in agreement with the dimension of the minimal basis for colour-ordered amplitudes. The kinematic factor $\mathcal{F}_{\ell}(\sigma, \alpha(i))$ is given by

$$
\mathcal{F}_{\ell}\left(\sigma, \alpha_{i}\right)=-s_{1 \alpha_{i}}-\sum_{\sigma_{j} \in \bar{\sigma}^{i} \cap\left(\underline{\alpha}_{i} \cup \beta\right)} s_{\alpha_{i} \sigma_{j}}-\left\{\begin{array}{ll}
-s_{\ell \alpha_{1} \ldots \alpha_{i-1}} & \text { if } \sigma_{\alpha_{i-1}}^{-1}<\sigma_{\alpha_{i}}^{-1}<\sigma_{\alpha_{i+1}}^{-1} \\
s_{\ell \alpha_{1} \ldots \alpha_{i}} & \text { if } \sigma_{\alpha_{i-1}}^{-1}>\sigma_{\alpha_{i}}^{-1}>\sigma_{\alpha_{i+1}}^{-1} \\
s_{\ell \alpha_{i}}+\sum_{\alpha_{j} \in \bar{\alpha}^{i}} s_{\alpha_{i} \alpha_{j}} & \text { if } \sigma_{\alpha_{i}}^{-1}>\sigma_{\alpha_{i-1}}^{-1}, \sigma_{\alpha_{i+1}}^{-1} \\
0 & \text { else }
\end{array}\right\} .
$$

Here by $\sigma_{\alpha(i)}^{-1}$ we denote the position of leg $\alpha(i)$ in the set $\sigma$, and $\bar{\sigma}^{i}$ and $\underline{\sigma}_{i}$ are the subsets of $\sigma$ comprising the elements that precede or follow $\alpha(i)$, respectively. The special cases for non-existent elements of $\alpha$ as follows

$$
\sigma_{\alpha(0)}^{-1}:=\sigma_{\alpha(2)}^{-1}, \quad \sigma_{\alpha(|\alpha|+1)}^{-1}:=0 .
$$

Here we have adapted the expression given in [1] to render the dependence on legs $\ell$ and $-\ell$ explicit. Other colour-ordered amplitudes are mapped to the minimal basis by a combination of Kleiss-Kuijf relations [3, 75] and the monodromy relation given above. Note that although this solution's denominators are not divergent in the forward limit, this is not true for the entirety of the momentum kernel.

\subsection{Forward limit of the momentum kernel}

In the forward limit where $q_{1}=-q_{2}=\ell$ so that $p_{1}+\cdots+p_{n}=0$, the momentum-kernel relation (3.8) is a polynomial of degree $n$ in $\ell$ of degree at most linear in each $\ell \cdot p_{i}$ with $1 \leq i \leq n$,

$$
\mathcal{S}(\ell)_{\sigma, \beta}:=\mathcal{S}[\sigma(1, \ldots, n) \mid \beta(1, \ldots, n)]_{\ell}=\prod_{i=1}^{n}\left(\ell \cdot p_{\sigma(i)}+\Theta_{i}(\sigma, \beta)\right),
$$

where $\Theta_{i}(\sigma, \beta)$ is defined in (3.9), and it is important that this quantity does not depend on the momentum $\ell$. Since in the forward limit the reference momentum is $\ell$ and permutations are acting on all the $n$ other external legs, we use a short-hand matrix notation $\mathcal{S}(\ell)_{\sigma, \beta}$.

In the forward limit, we find that due to the kinematical constraint $p_{1}+\cdots+p_{n}=0$ the momentum kernel degenerates, and its rank decreases. For massless momenta $p_{i}^{2}=0$ we computed numerically that

$$
\operatorname{dim} \operatorname{Ker}(\mathcal{S}(\ell))=(n-3) !\left(n^{2}-2 n+2\right)=(n-1) !+(n-2) !+2(n-3) !,
$$

see table 1. Moreover, we find the same dimension for the forward limit of the full momentum kernel in string theory

$$
\mathcal{S}_{\alpha^{\prime}}(\ell)=\prod_{i=1}^{n} \sin \left(\alpha^{\prime}\left(\ell \cdot p_{i}+\Theta_{i}(\sigma, \beta)\right)\right) .
$$




\begin{tabular}{|l|llllll|l|}
\hline $\mathrm{n}$ & 4 & 5 & 6 & 7 & 8 & 9 & generic $n$ \\
\hline$\left.\operatorname{dim} \operatorname{Ker} \mathcal{S}_{n+2}\right|_{q}$ & 6 & 24 & 120 & 720 & 5040 & 40320 & $(n-1) !$ \\
$\operatorname{dim} \operatorname{Ker} \mathcal{S}(\ell)(3.21)$ & 10 & 34 & 156 & 888 & 6000 & 46800 & $(n-3) !\left(n^{2}-2 n+2\right)$ \\
$\operatorname{dim} \operatorname{Ker} \mathcal{S}(3.26)$ or $(3.27)$ & 2 & 4 & 12 & 48 & - & - & $2(n-3) !$ \\
\hline
\end{tabular}

Table 1. Dimension of the kernel of the momentum kernel at $n+2$ points and in the forward limit. The first line gives the dimension of the kernel before taking the forward limit. We have numerically checked the rank for $n=4,5,6$ and 7. Since dim Ker $(3.21) /(n-3)$ ! cannot be more than a quadratic polynomial, three numerical points are enough to confirm the formula.

We propose the following interpretation. There are $(n+2)$ ! different orderings of the amplitudes including the permutations of the special forward legs $\ell$ and $-\ell$. In the forward limit some monodromy transformations vanish and leave more independent amplitudes characterised by the position of these special legs. Before taking the forward limit one expresses all colour-ordered amplitudes as a linear combination of elements in the basis composed of $A_{n+2}^{\text {tree }}\left(q_{1}, p_{1}, \alpha, q_{2}\right)$, where $\alpha \in \mathfrak{S}_{n-1}$ is the set of permutations of $n-1$ elements. In the forward limit this basis is composed of the single cuts of planar one-loop graphs

$$
B_{n-1}(\alpha):=\hat{A}_{n+2}^{\text {tree }}\left(\ell, p_{1}, \alpha,-\ell\right), \quad \alpha \in \mathfrak{S}_{n-1} .
$$

Normally, for any choice of three momenta $k_{1}, k_{2}$ and $k_{3}$ amongst $\left\{q_{1}, q_{2}, p_{1}, \ldots, p_{n}\right\}$, any colour-ordered tree amplitude $A_{n+2}^{\text {tree }}\left(k_{1}, \alpha, k_{2}, \beta, k_{3}, \gamma\right)$, where the disjoint union of the permutations $\alpha, \beta$ and $\gamma$ runs over the permutation of $n-1$ momenta $\mathfrak{S}_{n-1}$, can be expanded in the minimal basis $A_{n+2}^{\text {tree }}\left(k_{1}, k_{2}, \sigma, k_{3}\right)$, using a combination of Kleiss-Kuijf relations [3, 75] and the BCJ mapping given in [1, eq. (4.22)].

In the forward limit one needs to make the forward momenta special, and the choice of the three fixed momentum affects the monodromy relations. Some forward tree amplitudes become independent, depending whether the forward loop momenta are fixed or not. This is due to the vanishing of some coefficients in the monodromy relations. This happens when the BCJ map in [1, eq. (4.22)] develops a pole. The following two classes of regularised forward tree amplitudes cannot be related by a BCJ transformation to the element of basis $(3.24)$

$$
\begin{array}{ll}
B_{n-2}(\alpha):=\hat{A}_{n+2}^{\text {tree }}\left(\ell, p_{1}, \alpha,-\ell, p_{n}\right), & \alpha \in \mathfrak{S}_{n-2}, \\
B_{n-3}^{1}(\alpha):=\hat{A}_{n+2}^{\text {tree }}\left(p_{1}, p_{2}, \alpha, \ell,-\ell, p_{n}\right), & \alpha \in \mathfrak{S}_{n-3}, \\
B_{n-3}^{2}(\alpha):=\hat{A}_{n+2}^{\text {tree }}\left(p_{1}, p_{2}, \alpha,-\ell, \ell, p_{n}\right), & \alpha \in \mathfrak{S}_{n-3},
\end{array}
$$

There are $(n-1)$ ! elements in eq. (3.24), $(n-2)$ ! elements in eq. (3.25a) and $2(n-3)$ ! elements in eq. (3.25b). The sum of these dimensions equals to the one given in eq. (3.22). Notice that all the forward amplitudes in the sets $B_{n-1}(\alpha), B_{n-3}^{1}(\alpha$,$) and B_{n-3}^{2}(\alpha)$ arise from the single cut of planar one-loop amplitudes. The amplitudes in the set $B_{n-2}(\alpha)$ arise from the single cut of non-planar one-loop amplitudes.

The dimension in eq. (3.22) provides an upper bound on the number of partial oneloop amplitudes. For purely gluonic amplitudes in QCD and $\mathcal{N}=4$ super-Yang-Mills 
amplitudes at one loop, the planar cuts are enough. We can always consider single cuts that can be expressed on the minimal basis (3.24). For amplitudes with multiple flavoured matter particles in the fundamental representation, it is not yet entirely clear if non-planar cuts may be avoided as well. It would be interesting to determine the interplay with colour-kinematics duality for fundamental matter $[10,13]$ and understand if some single cuts need to be expressed in the basis (3.25a)-(3.25b). For instance, the Kleiss-Kuijf relations $[3,10,13,75]$ on non-planar cuts involve the planar cuts in $(3.25 \mathrm{~b})$. We note, as well, that the monodromy relations at higher-loop mix planar and non-planar cuts.

For the amplitude relation (3.10) the momentum kernel is

$$
\mathcal{S}[-\ell, 2, \ldots, n \mid \beta(-\ell, 2, \ldots, n)]_{\ell}=\left(-\ell^{2}-\sum_{i=2}^{n} \ell \cdot p_{i} \theta(1, i)\right) \prod_{i=2}^{n}\left(\ell \cdot p_{i}+\sum_{j>i}^{n} p_{i} \cdot p_{j} \theta(i, j)\right)
$$

and for the relations (3.11) the momentum kernel is

$$
\begin{aligned}
\mathcal{S}[\ell,-\ell, 3, \ldots, n \mid \beta(\ell,-\ell, 3, \ldots, n)]_{p_{1}}=\prod_{i=3}^{n}\left(p_{1} \cdot p_{i}+\sum_{j>i}^{n} p_{i} \cdot p_{j} \theta(i, j)\right) \\
\times\left(p_{1} \cdot \ell-\ell \cdot \ell \theta(1,2)+\sum_{j=3}^{n} \ell \cdot p_{j} \theta(1, j)\right)\left(-p_{1} \cdot \ell-\sum_{j=3}^{n} \ell \cdot p_{j} \theta(2, j)\right) .
\end{aligned}
$$

We recall that $\theta(i, j)$ is defined below eq. (3.9). We find that both these momentum kernel have dimension $2(n-3)$ !. This is the dimension of the basis of amplitudes in $B_{n-3}^{1}(\alpha)$ and $B_{n-3}^{2}(\alpha)$ in (3.25b).

\subsection{Monodromy relations and one-loop coefficients}

The monodromy relations imply that we can express the forward tree amplitudes in the minimal basis of forward tree amplitudes $\mathcal{B}=\left\{B_{r}^{\text {tree }}(\ell)\right\}$

$$
\hat{A}_{n+2}^{\text {tree }}\left(\sigma\left(\ell, p_{1}, \ldots, p_{n},-\ell\right)\right)=\sum_{r=1}^{\operatorname{dim}(\mathcal{B})} c_{r}^{\sigma}(\ell) B_{r}^{\text {tree }}(\ell) .
$$

The coefficients $c_{r}^{\sigma}(\ell)$ are explicitly known by solving the solving the monodromy relations (cf. [1, eq. (4.22)]).

The dimension of the kernel of the momentum kernel $\mathcal{S}(\ell)$ in (3.21) gives an upper bound on the dimension of the minimal basis $\operatorname{dim}(\mathcal{B}) \leq(n-3) !\left(n^{2}-2 n+2\right)$. The forward tree amplitudes $\hat{A}_{n+2}^{\text {tree }}\left(\ell, \sigma\left(p_{1}, \ldots, p_{n}\right),-\ell\right)$ can be expressed in the basis (3.24) of dimension $(n-1)$ !. This is enough for the single cuts of planar one-loop amplitudes.

We have explained in section 2.1 that the forward tree amplitudes contain all the information about the irreducible one-loop numerators $\Delta_{i_{1}, \ldots, i_{r}}(\ell)$. Therefore the number of independent one-loop irreducible numerators is given by the number of independent numerators in the minimal basis of forward tree amplitudes. Each of the numerators contains a one-loop integral coefficient that is non-spurious. So far our analysis provides an upper bound on the number of one-loop integral coefficients, it would be interesting to refine this analysis to obtain the optimal number of kinematically independent coefficients along the lines of $[76,77]$. 


\section{Field-theory monodromy relations from string theory}

In this section, we turn to the one-loop string-theory monodromies of [20] and their fieldtheory incarnation [21, 22], see also [78].

In the previous sections we have studied the kinematic relations on single-cuts obtained from the monodromies of forward tree-amplitudes. In section 4.1 we re-derive the forwardtree monodromies starting from the string-theory loop-monodromies. In section 4.2, we describe an important consequence of the string theory monodromies concerning the colourkinematic duality. First we explain how they actually give exact information about integrands even before integration. We do this as a first step toward a better control over the ambiguities in the labelling of the loop momentum when considering integration-by-parts identities [79-82]. We then illustrate this point by explaining how the BCJ relations are compatible with these field-theory monodromy relations obtained from string theory.

\subsection{Loop integrand monodromy versus forward-amplitude monodromies}

The field-theory limit of the integrand relations in string theory leads to the relations that were previously derived using field-theory techniques in [21, 22]. The first-order stringtheory contributions are discussed in detail in appendix B.

The one-loop fundamental monodromy relations between planar and non-planar open string integrands, $\mathcal{A}\left(p_{i_{1}}, \ldots, p_{i_{r}}\right)$ and $\mathcal{A}\left(p_{i_{1}}, \ldots, p_{i_{r}} \mid p_{i_{1}}, \ldots, p_{i_{s}}\right)$ reads

$$
\sum_{i=1}^{n-1} e^{i \pi \alpha^{\prime} p_{1} \cdot\left(\sum_{j=1}^{i} p_{j}\right)} \mathcal{A}(p_{2}, \ldots, \underbrace{p_{1}}_{\text {position } i}, \ldots, p_{n})=e^{-i \pi \alpha^{\prime} \ell \cdot p_{1}} \mathcal{A}\left(p_{2}, \ldots, p_{n} \mid p_{1}\right) .
$$

It was argued in [20] at the first order in $\alpha^{\prime}$ these relationship lead to the one-loop planar integrand relations in field theory

$$
\sum_{i=1}^{n-1} p_{1} \cdot\left(\ell+\sum_{j=1}^{i} p_{j}\right) \mathcal{I}(p_{2}, \ldots, \underbrace{p_{1}}_{\text {position }}, \ldots, p_{n}) \approx 0 .
$$

The notation " $\approx 0$ " means that, in field theory, the relations are valid up to contributions that vanish upon integration of the loop momentum [21, 22]. We show in section 4.2.1 that the string-theoretic construction actually determines the form of the terms in the righthand side of eq. (4.2). This implies that the relations can be thought of as exact integrand relationships.

At this stage, eq. (4.2) seems different from the forward tree monodromy relation (3.17a). The latter involves $n$ terms, whereas the former contains only $n-1$. So, first of all, we need to show how to relate both constructions.

The derivation of the monodromy relations in [20] was based on applying Cauchy's theorem to the open-string integrand in a loop momentum representation (crucial for holomorphy). We shall use the example of a five-point amplitude where 1 is circulated around the worldsheet boundary. The contour we study is pictured in the left-hand side of figure 2 .

At this point, strictly speaking we depart from the computation of [20]. We will follow the same reasoning, but applied to the single-cut of the one-loop amplitude, instead of 

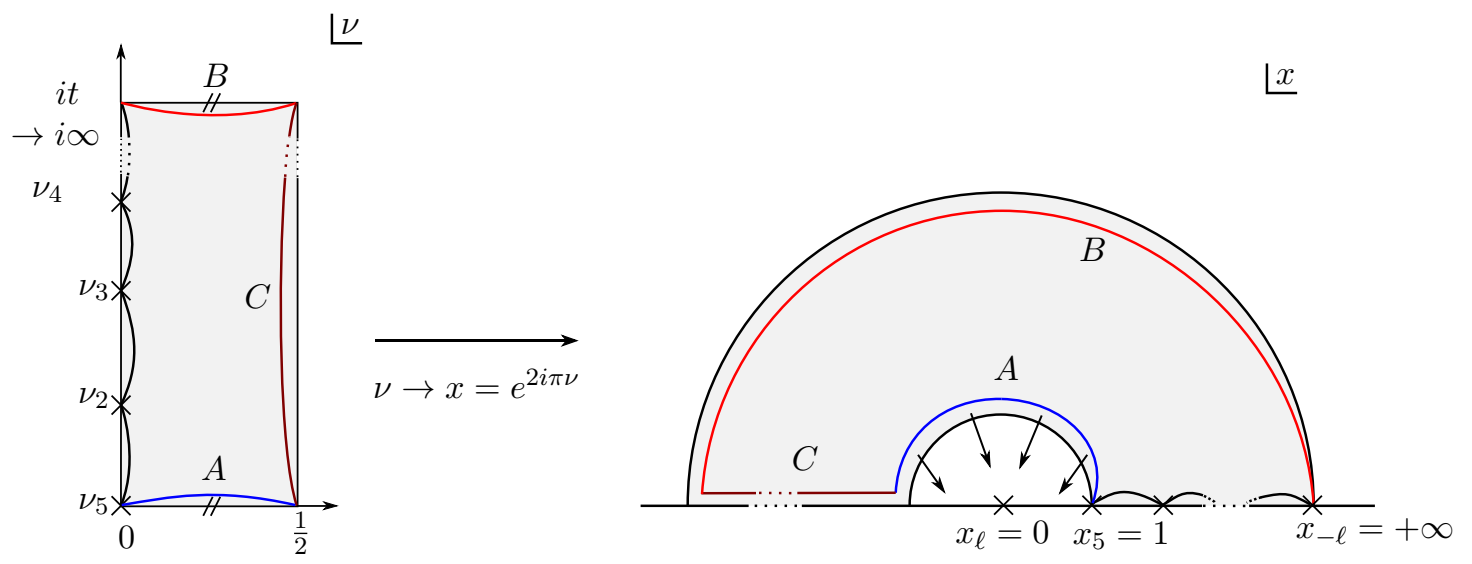

Figure 2. Mapping of the annulus to the plane with $x=e^{2 i \pi \nu}$ in the $t \rightarrow \infty$ limit. In the reduction of the blue contour onto the real axis, a singularity at $x=0$ is encountered.

the full string amplitude. These were defined in section 2 by the insertion of a $\delta^{(+)}\left(\ell^{2}\right)$ operator. We proved there that this induces the annulus to become an infinitely long strip. Via the exponential map $\nu \mapsto x=\exp (2 i \pi \nu)$, we send the strip to a portion of the upperhalf plane, as shown in figure 2. The segments of the integration contour are therefore also mapped on the upper-half plane.

The substance of the original monodromy-relations came from the vertical integration contours. In the present five-point planar example in eq. (4.1), the $\Re \mathrm{e}(\nu)=0$ contour gives rise to $\left(p_{i} \cdot p_{j}\right)$-type phases in contrast with the $\Re \mathrm{e}(\nu)=1 / 2$ contour $C$, which gives phases dependent on the loop momentum. However, since we now consider the monodromies of the single cut integrand, i.e. with the $\delta^{(+)}\left(\ell^{2}\right)$ function inserted, the boundary terms have a different fate than in [20].

\subsubsection{String-theoretic boundary terms}

The original string theory monodromies were based on discarding the $A$ and $B$ contours. This was justified because the two integrals only differ by a shift in the loop momentum. Namely, denoting $\mathcal{I}(\ell)$ the integrand of the string amplitude $\mathcal{A}$ being integrated along the closed contour, it was shown in [20] that

$$
\left.\mathcal{I}(\ell)\right|_{A}=\left.\mathcal{I}\left(\ell+p_{1}\right)\right|_{B}
$$

These terms vanish after integration over the loop momentum. We explicitly checked in [20] for $\mathcal{N}=4$ super-Yang-Mills that the numerators produced by the loop-momentum prefactors in eq. (4.2) cancel box propagators pairwise and produce six triangles, which cancel pairwise after a shift of the loop momentum.

Disregarding this cancellation, the application of Cauchy's theorem on the closed contour of figure 2 actually says that the left-hand side of eq. (4.2) is given by the difference of two terms that differ only by a shift in the loop momentum. That property obviously descends to the field-theory limit $\alpha^{\prime} \rightarrow 0$. We study the consequence of this fact later in section 4.2.1. 


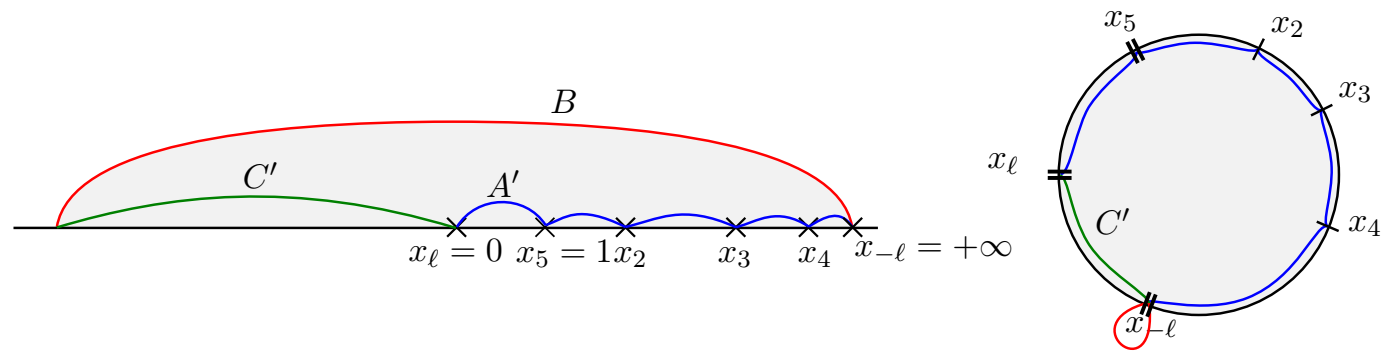

Figure 3. The right-hand side integration contour of figure 2 is equivalent to the one on the left-hand side here, which is equivalently represented in a disk picture on the right.

Note that the loop momentum arising in the string amplitude in (2.11) has a global definition, given by the integral along the $a$-cycle [62]

$$
\ell_{a}:=\oint_{a} \partial X=\int_{0}^{\frac{1}{2}} \frac{\partial X}{\partial \nu} d \nu
$$

with a similar definition at higher genus order. As was emphasised in [20], this means the string-theory limit induces a global definition of the loop momentum across all the Feynman graphs produced in this limit. This phenomenon is also observed in ambitwistorstring constructions [59-61].

\subsubsection{Recovering the single-cut monodromy relation}

To finish connecting the two monodromy relations (3.17a) and (4.2), we need to deform the contour of figure 2 down to the real axis. No singularity is present at $x=-1$, while one is present at 0 and the contours $A$ and $C$ can be turned into the contours $A^{\prime}$ and $C^{\prime}$ of figure 3. The contour $B$ vanishes as a consequence of onshellness and momentum conservation (which guarantees $\mathrm{SL}(2, \mathbb{R})$-invariance of the whole integral). This actually tells us that we are truly on the projective line and that the contour $C^{\prime}$ connects the puncture at $x=0$ and $x=+\infty$ as shown in the disk representation on the right-hand side of figure 2.

Notice that two additional states with momentum $\pm \ell$ appear at $x=0,+\infty$. This intuitive fact is easily derived by inspection of the degeneration of the one-loop KobaNielsen factor of string theory when $t \rightarrow 0$. In essence, such a computation is exposed in [59-61].

What we have shown so far is that, starting from a five-point amplitude (the example used here), we obtained five blue contours in figure 3 that correspond to the five terms from the circulation of the vertex operator 1 in the fundamental monodromy relation (3.17a). However, the one-loop monodromy relation in (4.2) had only four terms. The resolution of this apparent contradiction goes as follows.

Our starting point was actually not the original string-theory monodromies, but the monodromies of the projected amplitude (2.13) with the insertion of the single-cut operator $\delta^{(+)}\left(\ell^{2}\right)$. This term does not affect the phases, but it does break the freedom to shift the loop momentum to cancel out the integral between the contours $A$ and $B$. Adding the onshellness and momentum conservation condition, we see that the contribution from the 

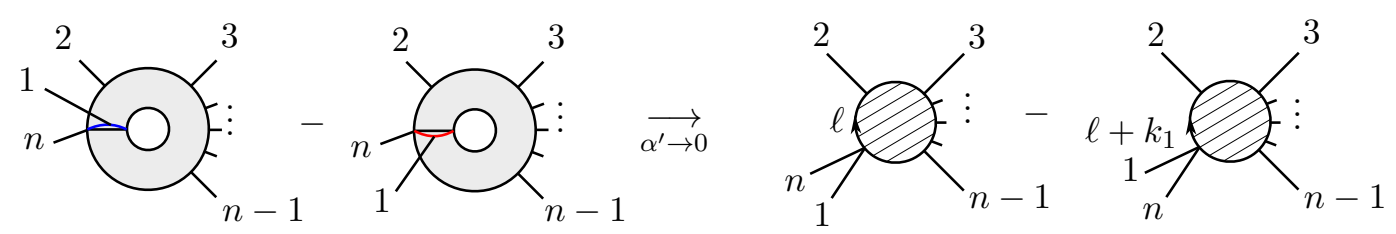

Figure 4. Left-hand side: boundary terms along contours $A$ and $B$ as defined in figure 2. Righthand side: conjectured corresponding types of one-loop graphs. Notice the loop-momentum shift.

contour $B$ drops out, and the contour $A$ gives an additional term that connects $x=0$ to $x=1$. One can then check that the phases of the momentum kernel, and the different terms match.

This concludes the proof of the correspondence between the string-theory monodromies and the forward monodromies.

\subsection{Dissecting the field theory limit of the string theory monodromies}

In this section we present two refinements of the string-theory monodromies. First, we comment on the inner structure of planar and non-planar diagrams arising from the string theory computation. Then we elaborate on the role of boundary terms from the stringtheoretic perspective. This will allow us to describe the BCJ-compatibility of the stringtheory monodromies in section 4.3.

To our knowledge these results are new and should be seen as an important refinement of the field-theory monodromies originally discovered in [21, 22].

\subsubsection{Role of boundary terms}

Let us come back to the interpretation of the string-theory monodromies in the light of the observation made in section 4.1.1. What we explained in eq. (4.3) and below was that the terms in the right-hand side of the string-theory monodromies needed to be of the form $F\left(\ell+p_{1}\right)-F(\ell)$.

Here we would like to motivate the following conjecture: the role of these terms is to accomodate for the fact that when the so-called "BCJ-moves" are done around a loop, a shift of the loop momentum needs to be made in the last move. This conjecture is motivated by the graphical argument that follows, and by the explicit examples in field theory at four and five points presented in section 4.3.

From before, we know that the terms that integrate to zero in the string theory monodromies come from the $A$ and $B$ contour integrals in figure 2. In the field-theory limit, we conjecture that these graphs need to appear as pinched contributions. Intuitively the reasoning is clear, and exposed in figure 4 . The drawing shows that the difference between the $A$ and $B$ contours results in graphs with a pinched propagator, that only differ by a shift of the loop momentum. In string theory, the shift arises because the loop momentum jumps when a puncture goes through the $a$-cycle on which it is defined, see eq. (4.4).

An actual extraction of the field theory limit to all loops, in the spirit of the "stringbased rules" [74, 83-86], would be interesting but very technical in nature and is outside of the scope of this paper. Such a computation should however prove two properties. 
First, that these graphs, coming from the $A$ and $B$ contours, appear at order $\alpha^{\prime}$. This is required by the fact that the integrand monodromy relations coming from string theory come at order $\mathcal{O}\left(\alpha^{\prime}\right)$. On dimensional grounds, it is clear that such pinched-terms contribute at order in $\alpha^{\prime}$ : the pinching of 1 and $n$ removes a propagator $1 /\left(\alpha^{\prime} p^{2}\right)$, therefore it contributes at order $\alpha^{\prime}$, even though it is a purely field-theoretic contribution.

Relatedly, the second part of the computation is to check that no 'triangle'-type graphs are generated in the process (i.e. graphs where a propagator of the form $1 /\left(p_{1} \cdot p_{n}\right)$. This is important because the momentum factor in the denominator has an inverse power of $\alpha^{\prime}$ that would make these terms contribute at $\mathcal{O}(1)$. Of course, because of the generic functional form $F\left(\ell+p_{1}\right)-F(\ell)$, these terms would still cancel after integration, even if present.

\subsubsection{Planar versus non-planar refinement}

In this section we clarify the connection of the string-theory monodromy relations (4.2) to the colour-kinematic duality. The result we present is that they can be rewritten as

$I^{b}(1,2, \ldots, n)+\sum_{i=2}^{n-1}\left[\left(\ell \cdot p_{1}\right) I^{b}(2, \ldots, i, 1, i+1, \ldots, n)+\left(p_{1} \cdot p_{2 \ldots i}\right) I(2, \ldots, i, 1, i+1, \ldots, n)\right] \approx 0$,

where the $\approx$ sign indicates again that the relation is valid modulo terms of the form $F(\ell+$ $\left.p_{1}\right)-F(\ell)$ that integrate to zero. Let us explain this new form of the monodromy relations.

The "flattened" integrand $I^{b}$ is defined as follows. Start from the non-planar one-loop string integrand $\mathcal{I}(2, \ldots, n ; 1)$ with leg 1 on one boundary and the other states on the other boundary. The traditional way of obtaining the integrand monodromies is to use the $\mathrm{U}(1)$-decoupling relations of [45] to rewrite this non-planar contribution as a sum of planar contributions. The outcome are the integrand relations we talked about that involve only planar integrands.

Instead of doing this, we will use the antisymmetry of the cubic vertices of the graphs entering the non-planar integrand in order to write them in a planar fashion. The sum of these graphs, for a particular ordering $(2, \ldots, i, 1, i+1, n)$, constitutes the term we call $I^{b}(2, \ldots, i, 1, i+1, n)$. The existence of such a representation, involving only graphs with antisymmetric cubic vertices, can be justified using the string-based rules [74, 83-86]. From the string-theoretic perspective, the idea is that the integrand can always undergo a succession of integrations by parts which allows to reduce it to a sum of only trivalent graphs. (For more details on this, see the review of the worldline formalism [86].) This is related to the fact that in string theory, gauge invariance is enforced differently than with the usual Feynman rules and does not require contact terms but only BRST closure of the expressions [87].

The peculiarity of the graphs entering $I^{b}(2, \ldots, i, 1, i+1, n)$ is that the leg 1 , being attached to the other colour trace, can never belong to an external tree attached to the loop. This is obvious from the string-theoretic perspective as well: the regions of integration giving rise to such trees are those for which a pair of punctures $\nu_{i}$ and $\nu_{j}$ become 


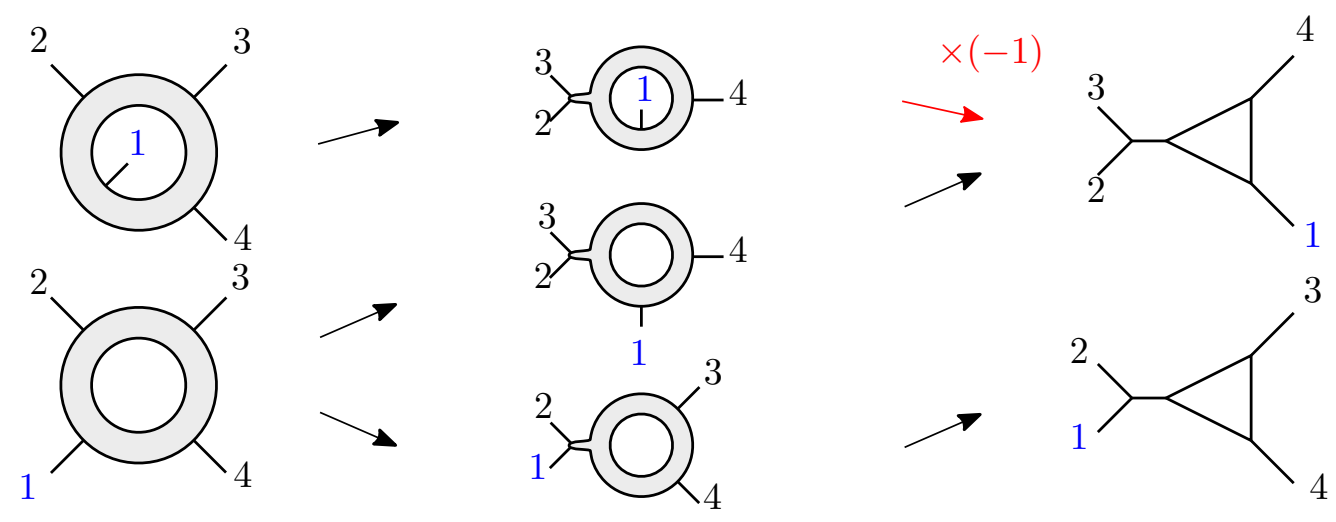

Figure 5. Triangles obtained by bringing $\nu_{2}$ close to $\nu_{3}$, or to $\nu_{1}$. The latter is not possible for the upper vertex configuration, in which $\nu_{1}$ is on the other boundary of the annulus.

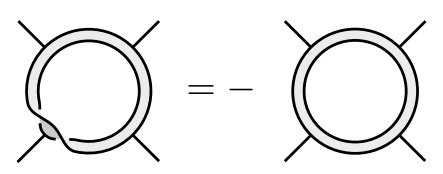

Figure 6. The antisymmetry of the three-point vertex converts non-planar contributions to planar.

infinitesimally close. This can not happen for the leg 1 , which is always on the opposite side of the annulus. This reasoning is illustrated in figure 5 .

This definition implies that

$$
\lim _{\alpha^{\prime} \rightarrow 0} \mathcal{I}(2, \ldots, n ; 1)=-\sum_{i=1}^{n} I^{b}(p_{2}, \ldots, \underbrace{p_{1}}_{\text {position } i}, \ldots, p_{n}),
$$

where it is understood that $I^{b}$ has no subtrees of the form of the lower one on the right-most side of figure 5. Since there is only one vertex to flip, this only results in a global sign.

Let us denote by $I(\sigma)$ the field-theory integrands coming from planar contributions, these with $\left(p_{i} \cdot p_{j}\right)$-type phases in the string-theory version and hence $\left(p_{i} \cdot p_{j}\right)$ factors in the field-theory limit. Similarly, we call $I^{b}(\sigma)$ the integrands coming from non-planar contributions but made planar using the antisymmetry of the three-point vertex for leg 1 , as in figure 6 . The important point we want to make is that these receive only phases of the form $\left(\ell \cdot p_{i}\right)$, or similar factors in the field-theory limit. Together with the previous considerations, this justifies the refined monodromy relation (4.5).

Below we dissect this relation and the role the boundary terms (which vanish after integration) in the case of a generic four-point amplitude.

\subsection{BCJ-compatibility of the string theory monodromies}

It was already observed in [21] that the integrand relations are satisfied at four points by $\mathcal{N}=4$ super-Yang-Mills. Shortly after [20], an $n$-point proof that BCJ representations satisfy the string theory monodromies was given in [24, 25]. For the sake of illustrating the refinements that were made in the previous section, we analyse the most general case of any massless theory that can be obtained as a limit of open-string theory. 


\subsubsection{Four-point case}

Let us consider the refined monodromies (4.5) in the four-point case:

$$
\begin{aligned}
\left(\ell \cdot p_{1}\right) I^{b}(1,2,3,4) & +\left(\ell \cdot p_{1}\right) I^{b}(2,1,3,4)+\left(\ell \cdot p_{1}\right) I^{b}(2,3,1,4) \\
& +\left(p_{1} \cdot p_{2}\right) I(2,1,3,4)+\left(p_{1} \cdot p_{23}\right) I(2,3,1,4) \approx 0 .
\end{aligned}
$$

We assume a cubic representation for the integrands composed of boxes, triangles and massive bubbles, i.e. the four-point topologies that do not integrate to zero in dimensional regularisation. Note again that $I^{b}(\sigma)$ lacks some of the diagrams present in $I(\sigma)$ - those with the special leg 1 in a massive corner of a triangle or a bubble represented in the first line of (4.8). We rewrite (4.7) by separating these contributions from the integrands of $I(2,1,3,4)$ and $I(2,3,1,4)$ in the second line and regroup the remaining pieces, $I^{b}(2,1,3,4)$ and $I^{b}(2,3,1,4)$ with the corresponding ones from the first line

$$
\begin{aligned}
\left(\ell \cdot p_{1}\right) I^{\mathrm{b}} & (1,2,3,4)+\left(\left(\ell+p_{2}\right) \cdot p_{1}\right) I^{\mathrm{b}}(2,1,3,4)+\left(\left(\ell+p_{23}\right) \cdot p_{1}\right) I^{\mathrm{b}}(2,3,1,4) \\
& +\left(p_{1} \cdot p_{2}\right)\left[I(2,1,3,4)-I^{\mathrm{b}}(2,1,3,4)\right]+\left(p_{1} \cdot p_{23}\right)\left[I(2,3,1,4)-I^{\mathrm{b}}(2,3,1,4)\right] \approx 0 .
\end{aligned}
$$

We can rewrite the kinematic coefficients in the first line as differences of propagators:

$$
\begin{aligned}
2\left(\ell \cdot p_{1}\right) & =\left(\ell+p_{1}\right)^{2}-\ell^{2}, & 2\left(\ell+p_{2}\right) \cdot p_{1}=\left(\ell+p_{12}\right)^{2}-\left(\ell+p_{2}\right)^{2}, \\
2\left(\ell+p_{23}\right) \cdot p_{1} & =\left(\ell-p_{4}\right)^{2}-\left(\ell-p_{14}\right)^{2} . &
\end{aligned}
$$

The cubic expansion for the monodromy relation (4.7) is then

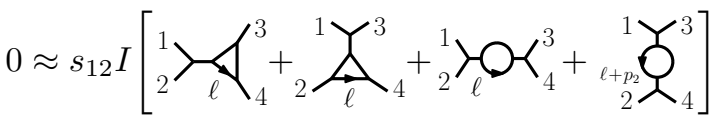

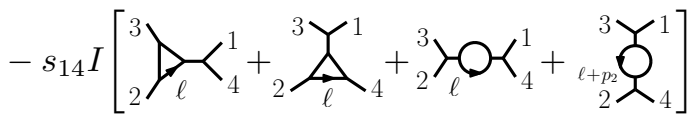

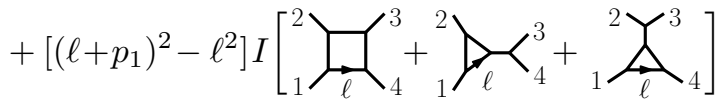

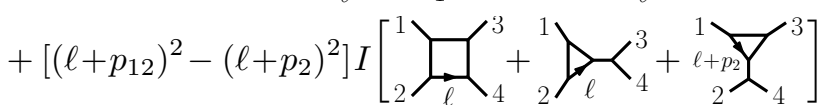

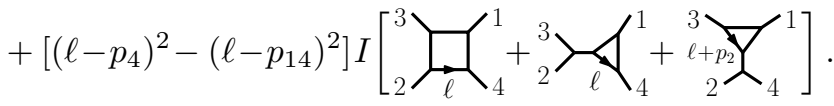

Remember that, although eq. (4.10) may seem gauge-dependent, its invariance is guaranteed by the U(1)-decoupling identity [74, eq. (6.4)],

$$
I^{\mathrm{b}}(1,2,3,4)+I^{\mathrm{b}}(2,1,3,4)+I^{\mathrm{b}}(2,3,1,4)=-I(2,3,4 ; 1) .
$$


Now we can collect terms with the same loop propagator structure. Some of them turn out to correspond to massless bubbles, so we omit them; the remaining ones are

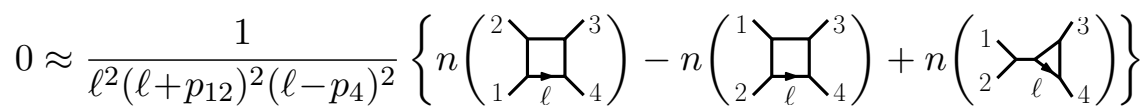

$$
\begin{aligned}
& \left.-\frac{1}{\left(\ell+p_{1}\right)^{2}\left(\ell+p_{12}\right)^{2}\left(\ell-p_{4}\right)^{2}} n\left({ }_{1}^{2}\right)-\Omega_{4}^{3}\right) \\
& \left.+\frac{1}{\ell^{2}\left(\ell+p_{2}\right)^{2}\left(\ell+p_{23}\right)^{2}}\left\{n\left({ }_{2}^{3}\right)_{\ell}{ }_{4}^{1}\right)-n\left({ }_{2}^{3} \gg_{\ell} \prec_{4}^{1}\right)\right\}
\end{aligned}
$$

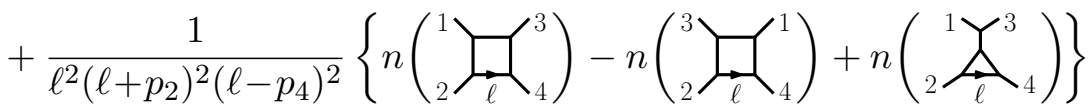

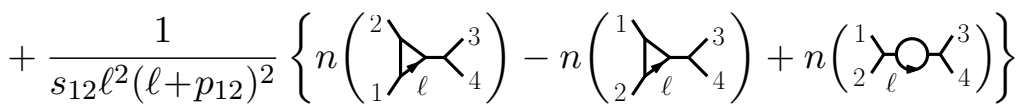

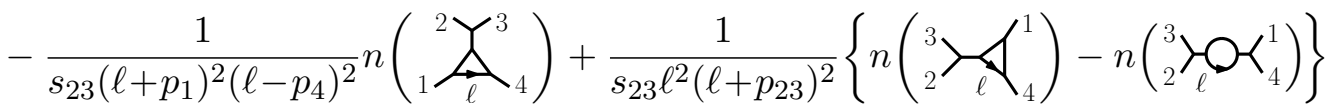

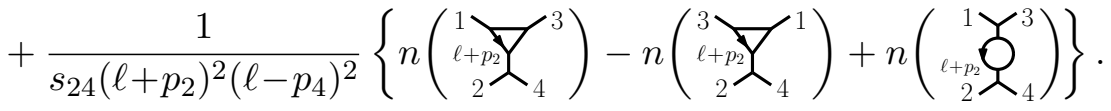

We immediately see that the cubic numerators organise themselves into triplets related by kinematic Jacobi identities. Note that in the second and fifth lines, one should shift the loop-momentum in one of the diagrams to reconstruct the correct Jacobi relations.

This is completely consistent with the exact-integrand relationship picture from string theory. In section 4.1 we explained that the right-hand side of eq. (4.7) has to be composed of terms of the form $F\left(\ell-p_{1}\right)-F(\ell)$, if the integrand relationship was written in a representation that is obtainable from the string-based rules. Here we see that only the terms which involve a loop momentum shift of the form $\ell \rightarrow \ell-p_{1}$ fail to constitute exact BCJ triplets.

From what we obtained in field theory, it seems that we can actually go further and constrain the form of string representations that would produce BCJ numerators.

Let us assume that we have one, and write down the refined monodromy relations, as in (4.12). All the BCJ triplets that do not involve a loop-momentum shift vanish, and we are left with those which involve a shift. They ought to equal the terms coming from the $A, B$ contours in the left-hand side; this constitutes a universal constraint on string-theoretic representations of BCJ numerators.

\subsubsection{Five-point case}

At higher points, distinct loop propagator structures appear with more than three cubic numerators. For example, the residue structure of the one-mass triangle $(2,3,451)$ is shared by the following numerators:

$$
\begin{aligned}
& \left.-\frac{1}{s_{45}\left(\ell+p_{1}\right)^{2}\left(\ell+p_{12}\right)^{2}\left(\ell-p_{45}\right)^{2}} n\left({ }_{1}^{2}\right)_{\ell}^{-C_{5}^{4}}\right) \\
& \left.+\frac{1}{\ell^{2}\left(\ell+p_{2}\right)^{2}\left(\ell+p_{23}\right)^{2}}\left\{\frac{1}{s_{45}} n\left({ }_{2}^{3}\right)_{\ell}^{-Z_{5}^{4}}\right)+\frac{s_{12}+s_{13}}{s_{23}}\left[\frac{1}{s_{45}} n\left({ }_{2}^{3} \nu_{\ell}^{1} r_{5}^{4}\right)+\frac{1}{s_{14}} n\left({ }_{2}^{3}\right\rangle_{\ell} r_{5}^{1}\right)\right]
\end{aligned}
$$




$$
\begin{aligned}
& \left.\left.+\frac{s_{12}+s_{13}+s_{14}}{s_{23}}\left[\frac{1}{s_{15}} n\left({ }_{2}^{3}\right\rangle_{\ell} Y_{5}^{4}\right)+\frac{1}{s_{14}} n({ }_{2}^{3} \overbrace{\ell} L_{1}^{4})\right]\right\} \\
& \left.=-\frac{1}{s_{45}\left(\ell+p_{1}\right)^{2}\left(\ell+p_{12}\right)^{2}\left(\ell-p_{45}\right)^{2}} n\left({ }_{1}^{2}\right)_{\ell}-_{5}^{3}\right) \\
& +\frac{1}{s_{45} \ell^{2}\left(\ell+p_{2}\right)^{2}\left(\ell+p_{23}\right)^{2}}\left\{n\left({ }_{2}^{3} \overrightarrow{-}_{\ell}^{1}-_{5}^{4}\right)-n\left({ }_{2}^{3} \nu_{\ell}^{1} T_{5}^{4}\right)\right\}
\end{aligned}
$$

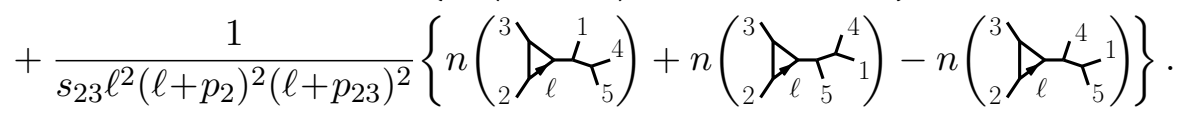

We performed the full five-point computation, and checked a few higher-point examples which support this claim. In these cases, we could verify that such expressions correspond to linear combinations of Jacobi identities.

This is consistent with the analysis of $[24,25]$ where it was shown that a colour-dual representation at $n$ points always satisfies the string-theory monodromies. However, no higher-point $n$-plets appeared in this analysis. This is possibly a consequence of their use of the multiperipheral representation of the colour factors of [75]. It would be interesting to understand this point in more detail.

\section{Discussion}

In this work we have studied the kinematic monodromy relations on single cuts of one-loop amplitudes in gauge theory. These relations are derived from the monodromy relations on the regularised forward tree amplitudes. We have explained that the regularisation does not affect the consequences of the monodromy relations for the irreducible one-loop numerators. We have checked that the monodromy relations are always satisfied by colourdual numerators, in agreement with the analysis of [24, 25].

The string-theoretic construction of [20] and the two-loop field-theory relations [23] show that higher-loop monodromy relations mix planar and non-planar sectors in interesting new ways. Indeed, the tree-level BCJ relations have already been used for a two-loop integrand calculation at full colour in [88]. Moreover, they have been seen to reduce the number of independent one-loop coefficients in [76, 77]. We look forward to these techniques converging to a systematic tool for multi-loop computations.

We have explained that the string-theory monodromy relations are exact relations, and their field-theory limit gives extra relations. This is due to the specific definition of the loop momenta in string theory. This approach gives a definite form for the terms that integrate to zero on the right-hand side of the field-theory relations. Precise control of the terms that are ambiguous in field-theoretic constructions will certainly be useful for deriving the consequences of the monodromy relations at loop orders in field theory. One other interesting application is the determination of well adapted classes of momentum-shifting identities for integration-by-parts identities [79-82]. For instance, it was found in [89] that a certain class of such contributions simplified the study of ultraviolet divergences in half-maximal supergravity. 
Finally, a comment on the co-rank of the momentum kernel. We found it to be given by $(n-1) !+(n-2) !+2(n-3) !$. Presumably, these relations can be interpreted in cohomological terms in the way of [90]. The counting differs from the number of solutions to the degenerate forward-tree scattering equations at one loop, which is $(n-1) !-2(n-2)$ ! [59, 72, 73, 91] and it would be really interesting to clarify the connection between the two quantities.

\section{Acknowledgments}

We would like to thank Simon Badger, Emil Bjerrum-Bohr, Simon Caron-Huot, John Joseph Carrasco, Michael B. Green, Julio Parra-Martinez, Radu Roiban and Oliver Schlotterer for valuable discussions.

AO is supported in part by the Marie-Curie FP7 grant 631370 . The work of PT is supported by STFC grant ST/L000385/1. The research of PV has received funding the ANR grant reference QST 12 BS05 003 01, and the CNRS grants PICS number 6430. PV is partially supported by a fellowship funded by the French Government at Churchill College, Cambridge. PV is grateful to the Mainz Institute for Theoretical Physics (MITP) for its hospitality and its partial support during the completion of this work. We would like to thanks Claude Duhr and the organisers of the workshop "LHC and the Standard Model: Physics and Tools" and the CERN theory division for the hospitality when completing this work.

\section{A Forward limit parametrisation}

If we start with on-shell momenta $p_{1}+p_{2}+\cdots+p_{n}=0$, we may deform the first two of them linearly in complex parameter $z$ in one of the following ways:

$$
\begin{array}{ll}
p_{1}(z)=\left(|1\rangle+z\left|\eta_{1}\right\rangle\right)[1 \mid, & p_{2}(z)=\left(|2\rangle+z\left|\eta_{2}\right\rangle\right)[2 \mid, \\
p_{1}(z)=\left(|1\rangle+z\left|\eta_{1}\right\rangle\right)[1 \mid, & p_{2}(z)=|2\rangle\left(\left[2 \mid+z\left[\tilde{\eta}_{2} \mid\right),\right.\right.
\end{array}
$$

such that $p_{i}^{2}(z)=0$ are preserved. We thus obtain $p_{1}(z)+p_{2}(z)+p_{3}+\cdots+p_{n} \equiv-z q$ for either $q=-\left|\eta_{1}\right\rangle\left[1|-| \eta_{2}\right\rangle\left[2 \mid\right.$ or $q=-\left|\eta_{1}\right\rangle[1|-| 2\rangle\left[\tilde{\eta}_{2} \mid\right.$. The next step is to define on-shell momenta $q_{2}=p_{n+1}(z)$ and $q_{1}=p_{n+2}(z)$ linearly dependent on $z$, such that

$$
p_{n+1}(z)+p_{n+2}(z)=z q, \quad p_{n+1}(z=0)=-\ell, \quad p_{n+2}(z=0)=\ell .
$$

We do this using the following generic solution for the Weyl spinors:

$$
\begin{aligned}
& \lambda_{\alpha}^{n+1}(z)=\left(\begin{array}{c}
\frac{-a\left(q^{1}-i q^{2}+b\left(q^{0}-q^{3}\right)\right)+z(q \cdot q)}{q^{0}+q^{3}+b\left(q^{1}+i q^{2}\right)} \\
a
\end{array}\right), \quad \tilde{\lambda}_{\dot{\alpha}}^{n+1}(z)=\left(\begin{array}{l}
1 \\
b
\end{array}\right), \\
& \lambda_{\alpha}^{n+2}(z)=\left(\begin{array}{c}
\frac{\left(q^{1}-i q^{2}+b\left(q^{0}-q^{3}\right)\right)\left(a+z\left(q^{1}+i q^{2}\right)\right)}{q^{0}+q^{3}+b\left(q^{1}+i q^{2}\right)} \\
-a-z\left(q^{1}+i q^{2}\right)
\end{array}\right), \quad \tilde{\lambda}_{\dot{\alpha}}^{n+2}(z)=\left(\begin{array}{c}
1 \\
a b-z\left(q^{0}+q^{3}\right) \\
a+z\left(q^{1}+i q^{2}\right)
\end{array}\right) .
\end{aligned}
$$


a)
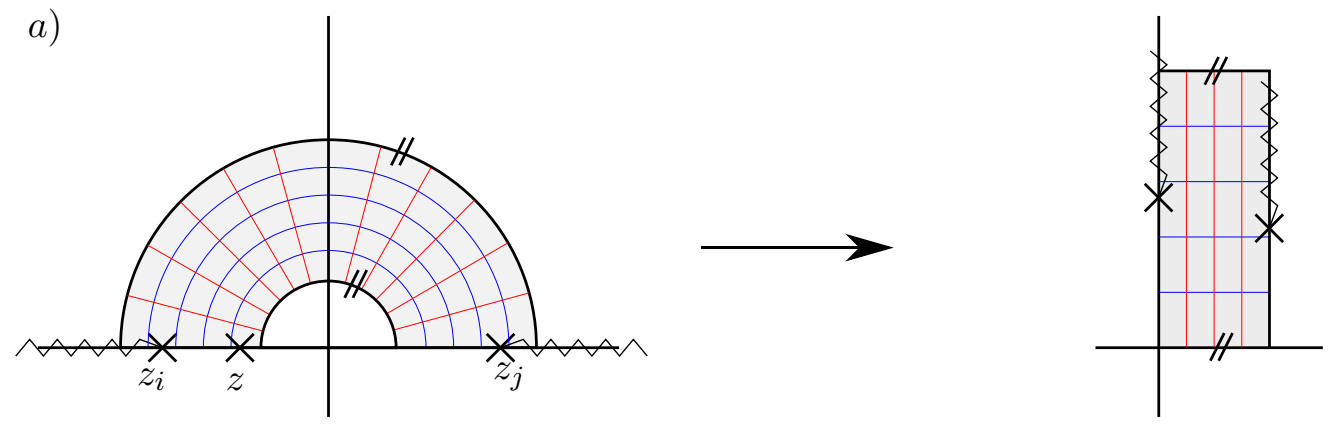

b)
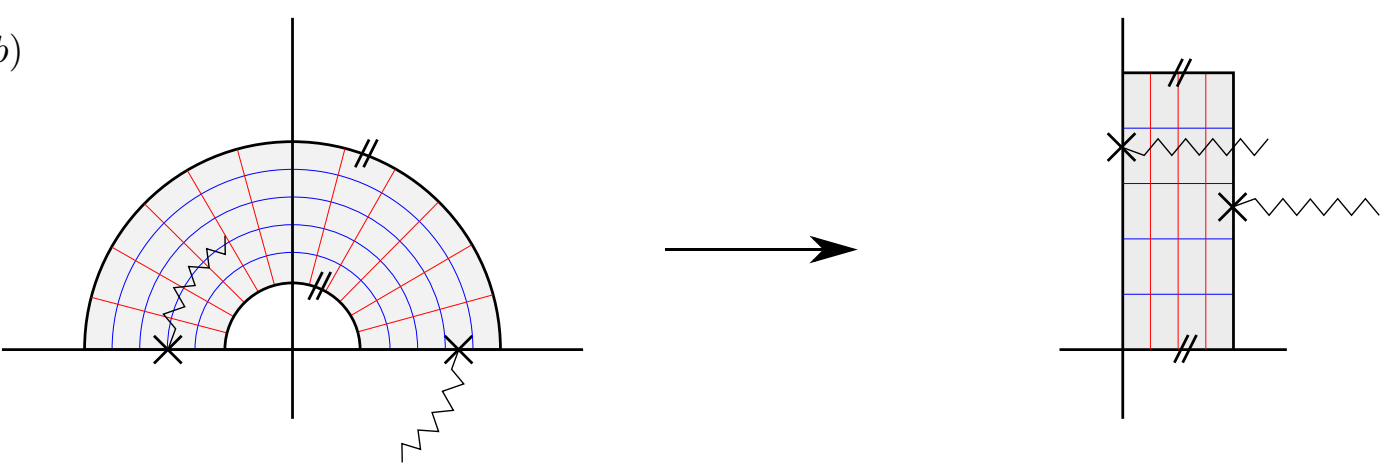

Figure 7. Determination of the complex logarithm on the annulus. The choice in [20] is $(a)$, whereas the convention of [31] is $(b)$.

\section{B One-loop monodromies in open string}

In this appendix we specify and develop some aspects of the open-string monodromies of [20]. We discuss how at one loop the original derivation [20] is related to the approach of the subsequent work [31].

\section{B.1 Prescription for monodromies and complex logarithm}

The most notable difference between the approaches of [20] and [31] is the choice of branch cuts for the Green's function. In [20] the branch cuts follow the boundary of the annulus and never cross its interior, as depicted in figure $7(a)$. This is an immediate generalisation of the choice made for the tree-level monodromy relations [4-6]. In [31] the cuts are chosen to go through the worldsheet in a maximal number of ways (see figure $7(b)$ ), thus requiring to deal with a variety of additional small contours of integration. Moreover, the definition of the logarithm in [20] does not require the introduction of bulk terms in the absence of closed-string operator insertions, to the contrary of the prescription used in [31]. Most importantly, converting one prescription to another leads to identical results.

\section{B.2 String theory one-loop monodromies at order $\alpha^{\prime}$}

To illustrate the general picture given above, we now expand the four-point relation given in [20] to the first order in $\alpha^{\prime}$ and find that the monodromy relations are satisfied. The presented analysis is rather similar to the check of the monodromy relation studied in [31]. 


\section{B.2.1 Planar four-point relation}

First, we check the validity of the planar four-point relation

$$
\mathcal{A}(1,2,3,4)+e^{i \pi \alpha^{\prime} p_{1} \cdot p_{2}} \mathcal{A}(2,1,3,4)+e^{i \pi \alpha^{\prime} p_{1} \cdot\left(p_{2}+p_{3}\right)} \mathcal{A}(2,3,1,4)+\mathcal{A}(2,3,4 \mid 1)\left[e^{-i \pi \alpha^{\prime} \ell \cdot p_{1}}\right]=0 .
$$

The planar amplitudes for four gauge bosons are given by

$$
\begin{aligned}
\mathcal{A}(\sigma(1,2,3,4))=t_{8} F^{4} \int d^{D} \ell & \int_{0}^{\infty} d t \int_{\Delta_{\sigma(1,2,3,4)}} d^{3} \nu \\
& \times e^{-\pi \alpha^{\prime} t \ell^{2}-2 i \pi \alpha^{\prime} \ell \cdot \sum_{i=1}^{4} p_{i} \nu_{i}} \prod_{1 \leq r<s \leq 4} e^{-\alpha^{\prime} p_{r} \cdot p_{s} G\left(\nu_{r}-\nu_{s}\right)} .
\end{aligned}
$$

The one-loop amplitude is proportional to the colour-ordered tree amplitude $t_{8} F^{4}$, where the $t_{8}$-tensor is defined in [92, appendix 9.A] and the Green's function is defined in eq. (2.12). ${ }^{4}$ To perform the $\alpha^{\prime}$-expansion, we integrate over the loop momentum and set to $D=10$ the critical dimension for open superstring theory ${ }^{5}$

$$
\mathcal{A}(\sigma(1,2,3,4))=t_{8} F^{4} \int_{0}^{\infty} \frac{d t}{\left(\alpha^{\prime} t\right)^{5}} \int_{\Delta_{\sigma(1,2,3,4)}} d^{3} \nu \prod_{1 \leq r<s \leq 4} e^{-\alpha^{\prime} p_{r} \cdot p_{s}\left(G\left(\nu_{r}-\nu_{s}\right)+\frac{\pi\left(\Im \mathrm{m}\left(\nu_{r}-\nu_{s}\right)\right)^{2}}{t}\right)},
$$

where the colour-ordered tree amplitude $A^{\text {tree }}(2,3,4 \mid 1)=t_{8} F^{4}$. The non-planar amplitude has a similar expression

$$
\begin{aligned}
\mathcal{A}(2,3,4 \mid 1)\left[e^{-i \pi \alpha^{\prime} \ell \cdot p_{1}}\right]= & -t_{8} F^{4} \int d^{D} \ell \int_{0}^{\infty} d t \int_{\Delta_{2,3,4 \mid 1}} d^{3} \nu \\
& \times e^{-\pi \alpha^{\prime} t \ell^{2}-2 i \pi \alpha^{\prime} \ell \cdot \sum_{i=1}^{4} p_{i} \nu_{i}-i \pi \alpha^{\prime} \ell \cdot p_{1}} \prod_{1 \leq r<s \leq 4} e^{-\alpha^{\prime} p_{r} \cdot p_{s} G\left(\nu_{r}-\nu_{s}\right)},
\end{aligned}
$$

with $\Re \mathrm{e}\left(\nu_{1}\right)=\frac{1}{2}$ and $\Re \mathrm{e}\left(\nu_{a}\right)=0$ with $a=2,3,4$. The overall sign arises from the orientation of boundary on which the vertex operator 1 is integrated. Integrating the loop momentum leads to

$$
\begin{aligned}
\mathcal{A}(2,3,4 \mid 1)\left[e^{-i \pi \alpha^{\prime} \ell \cdot p_{1}}\right]= & -t_{8} F^{4} \int_{0}^{\infty} \frac{d t}{\left(\alpha^{\prime} t\right)^{5}} \int_{\Delta_{2,3,4 \mid 1}} d^{3} \nu \\
& \times e^{\frac{i \pi \alpha^{\prime}}{t} p_{1} \cdot \sum_{i=1}^{4} p_{i} \nu_{i}} \prod_{1 \leq r<s \leq 4} e^{-\alpha^{\prime} p_{r} \cdot p_{s}\left(G\left(\nu_{r}-\nu_{s}\right)+\frac{\pi\left(\Im \mathrm{m}\left(\nu_{r}-\nu_{s}\right)\right)^{2}}{t}\right)} .
\end{aligned}
$$

\footnotetext{
${ }^{4}$ The integral is actually divergent from the $t \sim 0$ corresponding to the ultraviolet divergences of the planar open string graph. The one-loop open string amplitudes are finite only after the addition of the non-planar Moebius and Klein bottle. The amplitude relations are valid for any values of $t$ and are not affected by the ultraviolet behaviour of the individual graphs.

${ }^{5}$ We have used the identity

$$
\sum_{1 \leq r<s \leq n} p_{r} \cdot p_{s}\left(\Im \mathrm{m}\left(\nu_{r}-\nu_{s}\right)\right)^{2}=-\sum_{r, s=1}^{n} p_{r} \cdot p_{s} \Im \mathrm{m}\left(\nu_{r}\right) \Im \mathrm{m}\left(\nu_{s}\right)=-\left(\sum_{r=1}^{n} p_{r} \Im \mathrm{m}\left(\nu_{r}\right)\right)^{2}
$$

valid for $\sum_{r=1}^{n} p_{r}=0$. Notice that this identity does not require that $p_{r}^{2}=0$.
} 
The integration over $\Delta_{2,3,4 \mid 1}$ is defined as $0 \leq \Im \mathrm{m}\left(\nu_{2}\right) \leq \Im \mathrm{m}\left(\nu_{3}\right) \leq \Im \mathrm{m}\left(\nu_{4}\right)=$ it with $\Re \mathrm{e}\left(\nu_{i}\right)=0$ for $i=2,3,4$ and $\Re \mathrm{e}\left(\nu_{1}\right)=\frac{1}{2}$. Since

$$
G\left(\frac{1}{2}+\nu\right)=-\log \frac{\vartheta_{2}(\nu \mid i t)}{\vartheta_{1}^{\prime}(0)}=G(\nu)+\Delta G(\nu)
$$

where we have introduced

$$
\Delta G(\nu)=-\log \cot (\pi \nu)-4 \sum_{m \geq 0} \frac{q^{2 m+1}}{1-q^{2 m+1}} \frac{\cos (2 \pi(2 m+1) \nu)}{(2 m+1)} .
$$

Performing the expansion of (B.1) at the first order in $\alpha^{\prime}$, as a function of the proper time $t$, its integrand reads

$$
\begin{aligned}
i \pi \alpha^{\prime}\left(\int_{\Delta_{2,1,3,4}} d^{3} \nu p_{1} \cdot p_{2}-\int_{\Delta_{2,3,1,4}} d^{3} \nu p_{1} \cdot p_{4}\right) & -\int_{\Delta_{2,3,4 \mid 1}} d^{3} \nu \frac{\pi \alpha^{\prime} p_{1} \cdot \sum_{i=1}^{4} p_{i} \nu_{i}}{t} \\
& +\int_{\Delta_{1,2,3,4} \cup \Delta_{2,1,3,4} \cup \Delta_{2,3,1,4}} d^{3} \nu \mathcal{Q}-\mathcal{Q} \stackrel{?}{=} 0
\end{aligned}
$$

where we introduced the short-hand notation

$$
\mathcal{Q}=\sum_{1 \leq r<s \leq 4} p_{r} \cdot p_{s}\left(G\left(\nu_{r}-\nu_{s}\right)+\frac{\pi\left(\Im \mathrm{m}\left(\nu_{r}-\nu_{s}\right)\right)^{2}}{t}\right) .
$$

Using that a consequence of (B.7) allows to re-express the non-planar contribution as a sum of planar contributions

$$
\int_{\Delta_{2,3,4 \mid 1}} d^{3} \nu \mathcal{Q}=\int_{\Delta_{1,2,3,4} \cup \Delta_{2,1,3,4} \cup \Delta_{2,3,1,4}} d^{3} \nu(\mathcal{Q}-\Delta \mathcal{Q})
$$

where

$$
\Delta \mathcal{Q}=p_{1} \cdot p_{2} \Delta G\left(\nu_{1}-\nu_{2}\right)+p_{1} \cdot p_{3} \Delta G\left(\nu_{1}-\nu_{3}\right)+p_{1} \cdot p_{4} \Delta G\left(\nu_{1}-\nu_{4}\right) .
$$

The contributions from the phases compensate exactly each other because

$$
-\int_{\Delta_{2,3,4 \mid 1}} d^{3} \nu \frac{\pi \alpha^{\prime}}{t} p_{1} \cdot \sum_{i=1}^{4} p_{i} \nu_{i}=-\frac{\pi \alpha^{\prime} t^{3}}{6}\left(p_{1} \cdot p_{2}-p_{1} \cdot p_{4}\right)
$$

is the opposite of

$$
i \pi \alpha^{\prime}\left(\int_{\Delta_{2,1,3,4}} d^{3} \nu p_{1} \cdot p_{2}-\int_{\Delta_{2,3,1,4}} d^{3} \nu p_{1} \cdot p_{4}\right)=\frac{\pi \alpha^{\prime} t^{3}}{6}\left(p_{1} \cdot p_{2}-p_{1} \cdot p_{4}\right) .
$$

After cancellation of the integrals of $\mathcal{Q}$ using eq. (B.11), the relation (B.9) to prove becomes

$$
\int_{\Delta_{2,3,4 \mid 1}} d^{3} \nu \Delta \mathcal{Q} \stackrel{?}{=} 0
$$


We compute the integral by first evaluating the contributions to the $q$-expansion of $\Delta \mathcal{Q}$ from $q$-expansion in $\Delta G(\nu)$ in eq. (B.8) We find that setting $q=\exp (-2 \pi t)$

$$
\begin{aligned}
\left.\int_{\Delta_{2,3,4 \mid 1}} d^{3} \nu \Delta \mathcal{Q}\right|^{q-\exp }=i p_{1} \cdot p_{4}\{ & \frac{(\log q)^{2}}{64 \pi}+\frac{7(\log q) \zeta(3)}{32 \pi^{3}} \\
& +\frac{(\log q)^{2}\left(\operatorname{Li}_{2}(q)-\operatorname{Li}_{2}(-q)\right)}{16 \pi^{3}} \\
& \left.-\frac{\log q\left(\operatorname{Li}_{3}(q)-\operatorname{Li}_{3}(-q)\right)}{8 \pi^{3}}\right\} .
\end{aligned}
$$

To evaluate the zero mode, we have made use of the program HyperInt by Erik Panzer [93] to find

$$
\begin{aligned}
& \int_{\Delta_{2,3,4 \mid 1}} d^{3} \nu \log \left(\cot \pi\left(\nu_{1}-\nu_{2}\right)\right)=\frac{\log (q)\left(4 \operatorname{Li}_{3}(-q)-4 \operatorname{Li}_{3}(q)+\pi^{2} \log (q)+7 \zeta(3)\right)}{32 \pi^{3} i} \\
& \int_{\Delta_{2,3,4 \mid 1}} d^{3} \nu \log \left(\cot \pi\left(\nu_{1}-\nu_{3}\right)\right)=\frac{\log (q)\left(4 \operatorname{Li}_{3}(-q)-4 \operatorname{Li}_{3}(q)+\pi^{2} \log (q)+7 \zeta(3)\right)}{32 \pi^{3} i} \\
& \int_{\Delta_{2,3,4 \mid 1}} d^{3} \nu \log \left(\cot \pi\left(\nu_{4}-\nu_{1}\right)\right)=\frac{\left(4 \operatorname{Li}_{2}(-q)-4 \operatorname{Li}_{2}(q)+\pi^{2}\right) \log ^{2}(q)}{64 \pi^{3} i}
\end{aligned}
$$

Summing over these contributions leads to complete cancellation between the integral over the zero-mode part and the $q$-expansion in $\Delta \mathcal{Q}$, establishing that for all values of the proper time $t$

$$
\int_{\Delta_{2,3,4 \mid 1}} d^{3} \nu \Delta \mathcal{Q} \stackrel{\vee}{=} 0 .
$$

This completes the verification of the amplitude relation (B.1) to the first order in the $\alpha^{\prime}$-expansion.

Notice that the vanishing is satisfied for all values of the proper time $t$, and therefore there is no need of considering a regularisation of the ultraviolet divergence (for $t \sim 0$ ) of the annulus graph.

\section{B.2.2 Non-planar four-point relation}

The non-planar $n$-point monodromy relation is given by ${ }^{6}$

$$
\begin{aligned}
\mathcal{A}(1,2, \ldots, p \mid p+1, \ldots, n)+\sum_{i=2}^{p-1} e^{i \alpha^{\prime} \pi p_{1} \cdot p_{2} \ldots i} \mathcal{A}(2, \ldots, i, 1, i+1, \ldots, p \mid p+1, \ldots, n) & \\
- & \sum_{i=p}^{n}\left(e^{-i \alpha^{\prime} \pi p_{1} \cdot p_{i+1} \ldots n} \times \mathcal{A}(2, \ldots, p \mid p+1, \ldots, i, 1, i+1, \ldots, n)\left[e^{-i \pi \alpha^{\prime} \ell \cdot p_{1}}\right]\right)=0 .
\end{aligned}
$$

\footnotetext{
${ }^{6}$ We correct a sign mistake in [20] for the non-planar phases. The non-planar cuts were incorrectly determined to be downward cuts, while in fact they are upward cuts, as pictured in the right column of figure $7(a)$.
} 
We now wish to verify the following non-planar four-point relation:

$$
\begin{aligned}
\mathcal{A}(1,2 \mid 3,4)+e^{i \pi \alpha^{\prime} p_{1} \cdot\left(p_{3}+p_{4}\right)} \mathcal{A}(2 \mid 1,3,4)\left[e^{-i \pi \alpha^{\prime} \ell \cdot p_{1}}\right] & \\
& +e^{i \pi \alpha^{\prime} p_{1} \cdot p_{4}} \mathcal{A}(2 \mid 3,1,4)\left[e^{-i \pi \alpha^{\prime} \ell \cdot p_{1}}\right]+\mathcal{A}(2 \mid 3,4,1)\left[e^{-i \pi \alpha^{\prime} \ell \cdot p_{1}}\right]=0 .
\end{aligned}
$$

The first non-planar amplitude is given by

$$
\mathcal{A}(1,2 \mid 3,4)=t_{8} F^{4} \int d^{D} \ell \int_{0}^{\infty} d t \int_{\Delta_{1,2 \mid 3,4}} d^{3} \nu e^{-\pi \alpha^{\prime} t \ell^{2}-2 i \pi \alpha^{\prime} \ell \cdot \sum_{i=1}^{4} p_{i} \nu_{i}} \prod_{1 \leq r<s \leq 4} e^{-\alpha^{\prime} p_{r} \cdot p_{s} G\left(\nu_{r}-\nu_{s}\right)},
$$

where $\Delta_{12 \mid 34}$ is defined by $\nu_{2}=i t, 0 \leq \Im \mathrm{m}\left(\nu_{1}\right) \leq t$ and $0 \leq \Im \mathrm{m}\left(\nu_{4}\right) \leq \Im \mathrm{m}\left(\nu_{3}\right) \leq t$. We integrate over the loop momentum to get the expression

$$
\mathcal{A}(1,2 \mid 3,4)=t_{8} F^{4} \int_{0}^{\infty} \frac{d t}{\left(\alpha^{\prime} t\right)^{5}} \int_{\Delta_{1,2 \mid 3,4}} d^{3} \nu \prod_{1 \leq r<s \leq 4} e^{-\alpha^{\prime} p_{r} \cdot p_{s}\left(G\left(\nu_{r}-\nu_{s}\right)+\frac{\pi\left(\Im \mathrm{m}\left(\nu_{r}-\nu_{s}\right)\right)^{2}}{t}\right)} .
$$

The next amplitude is

$$
\begin{aligned}
\mathcal{A}(2 \mid 1,3,4)\left[e^{-i \pi \alpha^{\prime} \ell \cdot p_{1}}\right]=-t_{8} F^{4} \int d^{D} \ell \int_{0}^{\infty} d t \int_{\Delta_{2 \mid 1,3,4}} d^{3} \nu e^{-\pi \alpha^{\prime} t \ell^{2}-2 i \pi \alpha^{\prime} \ell \cdot \sum_{i=1}^{4} p_{i} \nu_{i}-\pi \alpha^{\prime} \ell \cdot p_{1}} & \left.\times \prod_{1 \leq r<s \leq 4} e^{-\alpha^{\prime} p_{r} \cdot p_{s} G\left(\nu_{r}-\nu_{s}\right)}, \quad \text { (B. } 23\right)
\end{aligned}
$$

where the overall sign comes from the contour of integration, and $\Delta_{2 \mid 1,3,4}$ is defined by $\nu_{2}=i t$ and $0 \leq \Im \mathrm{m}\left(\nu_{4}\right) \leq \Im \mathrm{m}\left(\nu_{3}\right) \leq \Im \mathrm{m}\left(\nu_{1}\right) \leq t$, with $\Re \mathrm{e}\left(\nu_{2}\right)=0$ and $\Re \mathrm{e}\left(\nu_{i}\right)=\frac{1}{2}$ for $i=1,3,4$. Equivalent definitions determine the amplitudes $\mathcal{A}(2 \mid 3,1,4)\left[e^{-i \pi \alpha^{\prime} \ell \cdot p_{1}}\right]$ and $\mathcal{A}(2 \mid 3,4,1)\left[e^{-i \pi \alpha^{\prime} \ell \cdot p_{1}}\right]$. Integrating over the loop momentum gives

$$
\begin{aligned}
\mathcal{A}(2 \mid 1,3,4)\left[e^{-i \pi \alpha^{\prime} \ell \cdot p_{1}}\right]=-t_{8} F^{4} \int_{0}^{\infty} \frac{d t}{\left(\alpha^{\prime} t\right)^{5}} \int_{\Delta_{2 \mid 1,3,4}} d^{3} \nu e^{\frac{i \pi \alpha^{\prime}}{t} p_{1} \cdot \sum_{i=1}^{4} p_{i} \nu_{i}} \\
\times \prod_{1 \leq r<s \leq 4} e^{-\alpha^{\prime} p_{r} \cdot p_{s}\left(G\left(\nu_{r}-\nu_{s}\right)+\frac{\pi\left(\Im \mathrm{m}\left(\nu_{r}-\nu_{s}\right)\right)^{2}}{t}\right)} .
\end{aligned}
$$

Like before, integrating out the loop momentum results in phase factors that can be explicitly computed at first order in $\alpha^{\prime}$

$$
\begin{aligned}
\int_{\Delta_{2 \mid 1,3,4} \cup \Delta_{2 \mid 3,1,4} \cup \Delta_{2 \mid 3,4,1}} d^{3} \nu \frac{\pi \alpha^{\prime} p_{1} \cdot \sum_{i=1}^{4} p_{i} \nu_{i}}{t} \\
+i \pi \alpha^{\prime}\left(\int_{\Delta_{2 \mid 1,3,4}} d^{3} \nu p_{1} \cdot p_{34}+\int_{\Delta_{2 \mid 3,4,1}} d^{3} \nu p_{1} \cdot p_{4}\right) \\
+\int_{\Delta_{12 \mid 34}} d^{3} \nu \mathcal{Q}-\int_{\Delta_{2 \mid 1,3,4} \cup \Delta_{2 \mid 3,1,4} \cup \Delta_{2 \mid 3,4,1}} d^{3} \nu \mathcal{Q} \stackrel{?}{=} 0,
\end{aligned}
$$

where $\mathcal{Q}$ has been introduced in eq. (B.10). We can rewrite this integral as

$$
\begin{aligned}
& \int_{\Delta_{2 \mid 1,3,4} \cup \Delta_{2 \mid 3,1,4} \cup \Delta_{2 \mid 3,4,1}} d^{3} \nu \frac{\pi \alpha^{\prime} p_{1} \cdot \sum_{i=1}^{4} p_{i} \nu_{i}}{t} \\
& +i \pi \alpha^{\prime}\left(\int_{\Delta_{2 \mid 1,3,4}} d^{3} \nu p_{1} \cdot p_{34}+\int_{\Delta_{2 \mid 3,1,4}} d^{3} \nu p_{1} \cdot p_{4}\right)-\int_{\Delta_{12 \mid 34}} d^{3} \nu \Delta \mathcal{Q} \stackrel{?}{=} 0,
\end{aligned}
$$


where $\Delta \mathcal{Q}$ is defined in eq. (B.12). We then find

$$
\begin{aligned}
\int_{\Delta_{2 \mid 1,3,4} \cup \Delta_{2 \mid 3,1,4} \cup \Delta_{2 \mid 3,4,1}} d^{3} \nu \frac{\pi \alpha^{\prime}}{t} p_{1} \cdot \sum_{i=1}^{4} p_{i} \nu_{i} & =\frac{\pi \alpha^{\prime} t^{3}}{6}\left(p_{1} \cdot p_{2}-p_{1} \cdot p_{4}\right), \\
\int_{\Delta_{2 \mid 1,3,4}} d^{3} \nu\left(i \pi \alpha^{\prime} p_{1} \cdot p_{34}\right) & =-\frac{\pi \alpha^{\prime} t^{3}}{6} p_{1} \cdot p_{2}, \\
\int_{\Delta_{2 \mid 3,1,4}} d^{3} \nu\left(i \pi \alpha^{\prime} p_{1} \cdot p_{4}\right) & =\frac{\pi \alpha^{\prime} t^{3}}{6} p_{1} \cdot p_{4},
\end{aligned}
$$

therefore we see cancellation of the phase factors in the first line of eq. (B.26). For the second line we find for the $q$-expansion of $\Delta \mathcal{Q}$

$$
\begin{aligned}
\left.\int_{\Delta_{1,2 \mid 3,4}} d^{3} \nu \Delta \mathcal{Q}\right|^{q-e x p}= & -i p_{1} \cdot p_{2} \frac{\log (q)^{2}}{64 \pi^{3}}\left(\frac{3}{2} \zeta(2)+\operatorname{Li}_{2}(q)-\operatorname{Li}_{2}(-q)\right) \\
& -i p_{1} \cdot p_{2} \frac{\log (q)}{64 \pi^{3}}\left(-\frac{7}{4} \zeta(3)+\operatorname{Li}_{3}(q)-\mathrm{Li}_{3}(-q)\right),
\end{aligned}
$$

which is cancelled against the constant terms from $\Delta \mathcal{Q}$. Hence we arrive at

$$
\int_{\Delta_{1,2 \mid 3,4}} d^{3} \nu \Delta \mathcal{Q} \stackrel{\vee}{=} 0,
$$

which proves the identity (B.26).

Open Access. This article is distributed under the terms of the Creative Commons Attribution License (CC-BY 4.0), which permits any use, distribution and reproduction in any medium, provided the original author(s) and source are credited.

\section{References}

[1] Z. Bern, J.J.M. Carrasco and H. Johansson, New relations for gauge-theory amplitudes, Phys. Rev. D 78 (2008) 085011 [arXiv: 0805. 3993] [InSPIRE].

[2] Z. Bern, J.J.M. Carrasco and H. Johansson, Perturbative quantum gravity as a double copy of gauge theory, Phys. Rev. Lett. 105 (2010) 061602 [arXiv:1004.0476] [INSPIRE].

[3] R. Kleiss and H. Kuijf, Multi-gluon cross-sections and five jet production at hadron colliders, Nucl. Phys. B 312 (1989) 616 [inSPIRE].

[4] N.E.J. Bjerrum-Bohr, P.H. Damgaard and P. Vanhove, Minimal basis for gauge theory amplitudes, Phys. Rev. Lett. 103 (2009) 161602 [arXiv:0907.1425] [INSPIRE].

[5] N.E.J. Bjerrum-Bohr, P.H. Damgaard, T. Sondergaard and P. Vanhove, Monodromy and jacobi-like relations for color-ordered amplitudes, JHEP 06 (2010) 003 [arXiv: 1003.2403] [INSPIRE].

[6] S. Stieberger, Open \& closed vs. pure open string disk amplitudes, arXiv:0907.2211 [INSPIRE].

[7] C.R. Mafra, O. Schlotterer and S. Stieberger, Explicit BCJ numerators from pure spinors, JHEP 07 (2011) 092 [arXiv:1104.5224] [INSPIRE]. 
[8] R. Monteiro and D. O'Connell, The kinematic algebra from the self-dual sector, JHEP $\mathbf{0 7}$ (2011) 007 [arXiv: 1105.2565] [INSPIRE].

[9] M. Chiodaroli, Q. Jin and R. Roiban, Color/kinematics duality for general abelian orbifolds of $N=4$ super Yang-Mills theory, JHEP 01 (2014) 152 [arXiv:1311.3600] [INSPIRE].

[10] H. Johansson and A. Ochirov, Pure gravities via color-kinematics duality for fundamental matter, JHEP 11 (2015) 046 [arXiv:1407.4772] [INSPIRE].

[11] M. Chiodaroli, M. Günaydin, H. Johansson and R. Roiban, Scattering amplitudes in $\mathcal{N}=2$ Maxwell-Einstein and Yang-Mills/Einstein supergravity, JHEP 01 (2015) 081 [arXiv:1408.0764] [INSPIRE].

[12] S. He, R. Monteiro and O. Schlotterer, String-inspired BCJ numerators for one-loop MHV amplitudes, JHEP 01 (2016) 171 [arXiv: 1507.06288] [INSPIRE].

[13] H. Johansson and A. Ochirov, Color-kinematics duality for QCD amplitudes, JHEP 01 (2016) 170 [arXiv: 1507.00332] [INSPIRE].

[14] G. Mogull and D. O'Connell, Overcoming obstacles to colour-kinematics duality at two loops, JHEP 12 (2015) 135 [arXiv: 1511.06652] [INSPIRE].

[15] H. Johansson, G. Kälin and G. Mogull, Two-loop supersymmetric QCD and half-maximal supergravity amplitudes, JHEP 09 (2017) 019 [arXiv: 1706.09381] [INSPIRE].

[16] M. Chiodaroli, M. Günaydin, H. Johansson and R. Roiban, Explicit formulae for Yang-Mills-Einstein amplitudes from the double copy, JHEP 07 (2017) 002 [arXiv: 1703.00421] [INSPIRE].

[17] Z. Bern, S. Davies and T. Dennen, The ultraviolet structure of half-maximal supergravity with matter multiplets at two and three loops, Phys. Rev. D 88 (2013) 065007 [arXiv:1305.4876] [INSPIRE].

[18] Z. Bern, S. Davies and T. Dennen, Enhanced ultraviolet cancellations in $\mathcal{N}=5$ supergravity at four loops, Phys. Rev. D 90 (2014) 105011 [arXiv:1409.3089] [INSPIRE].

[19] Z. Bern, J.J. Carrasco, W.-M. Chen, H. Johansson and R. Roiban, Gravity amplitudes as generalized double copies of gauge-theory amplitudes, Phys. Rev. Lett. 118 (2017) 181602 [arXiv: 1701.02519] [INSPIRE].

[20] P. Tourkine and P. Vanhove, Higher-loop amplitude monodromy relations in string and gauge theory, Phys. Rev. Lett. 117 (2016) 211601 [arXiv:1608.01665] [INSPIRE].

[21] R.H. Boels and R.S. Isermann, New relations for scattering amplitudes in Yang-Mills theory at loop level, Phys. Rev. D 85 (2012) 021701 [arXiv:1109.5888] [InSPIRE].

[22] R.H. Boels and R.S. Isermann, Yang-Mills amplitude relations at loop level from non-adjacent BCFW shifts, JHEP 03 (2012) 051 [arXiv:1110.4462] [INSPIRE].

[23] B. Feng, Y. Jia and R. Huang, Relations of loop partial amplitudes in gauge theory by unitarity cut method, Nucl. Phys. B 854 (2012) 243 [arXiv:1105. 0334] [INSPIRE].

[24] R.W. Brown and S.G. Naculich, Color-factor symmetry and BCJ relations for QCD amplitudes, JHEP 11 (2016) 060 [arXiv:1608.05291] [INSPIRE].

[25] R.W. Brown and S.G. Naculich, BCJ relations from a new symmetry of gauge-theory amplitudes, JHEP 10 (2016) 130 [arXiv:1608.04387] [INSPIRE].

[26] S. He and O. Schlotterer, New relations for gauge-theory and gravity amplitudes at loop level, Phys. Rev. Lett. 118 (2017) 161601 [arXiv:1612.00417] [INSPIRE]. 
[27] S. He, O. Schlotterer and Y. Zhang, New BCJ representations for one-loop amplitudes in gauge theories and gravity, arXiv:1706.00640 [INSPIRE].

[28] F. Cachazo, S. He and E.Y. Yuan, Scattering of massless particles in arbitrary dimensions, Phys. Rev. Lett. 113 (2014) 171601 [arXiv:1307.2199] [InSPIRE].

[29] F. Cachazo, S. He and E.Y. Yuan, Scattering of massless particles: scalars, gluons and gravitons, JHEP 07 (2014) 033 [arXiv:1309.0885] [INSPIRE].

[30] N.E.J. Bjerrum-Bohr, P.H. Damgaard, T. Sondergaard and P. Vanhove, The momentum kernel of gauge and gravity theories, JHEP 01 (2011) 001 [arXiv:1010.3933] [INSPIRE].

[31] S. Hohenegger and S. Stieberger, Monodromy relations in higher-loop string amplitudes, arXiv:1702.04963 [INSPIRE].

[32] R.P. Feynman, Quantum theory of gravitation, Acta Phys. Polon. 24 (1963) 697 [InSPIRE].

[33] R.P. Feynman, Closed loop and tree diagrams, in Selected papers of Richard Feynman, L.M. Brown ed., World Scientific, Singapore(1972).

[34] A. Brandhuber, B. Spence and G. Travaglini, From trees to loops and back, JHEP 01 (2006) 142 [hep-th/0510253] [INSPIRE].

[35] F. Cachazo, P. Svrček and E. Witten, MHV vertices and tree amplitudes in gauge theory, JHEP 09 (2004) 006 [hep-th/0403047] [INSPIRE].

[36] S. Catani, T. Gleisberg, F. Krauss, G. Rodrigo and J.-C. Winter, From loops to trees by-passing Feynman's theorem, JHEP 09 (2008) 065 [arXiv:0804.3170] [INSPIRE].

[37] I. Bierenbaum, S. Catani, P. Draggiotis and G. Rodrigo, A tree-loop duality relation at two loops and beyond, JHEP 10 (2010) 073 [arXiv:1007.0194] [INSPIRE].

[38] I. Bierenbaum, M. Czakon and A. Mitov, The singular behavior of one-loop massive QCD amplitudes with one external soft gluon, Nucl. Phys. B 856 (2012) 228 [arXiv:1107.4384] [INSPIRE].

[39] I. Bierenbaum, S. Buchta, P. Draggiotis, I. Malamos and G. Rodrigo, Tree-loop duality relation beyond simple poles, JHEP 03 (2013) 025 [arXiv:1211.5048] [INSPIRE].

[40] S. Buchta, G. Chachamis, P. Draggiotis, I. Malamos and G. Rodrigo, On the singular behaviour of scattering amplitudes in quantum field theory, JHEP 11 (2014) 014 [arXiv: 1405.7850] [INSPIRE].

[41] E.W. Nigel Glover and C. Williams, One-loop gluonic amplitudes from single unitarity cuts, JHEP 12 (2008) 067 [arXiv:0810.2964] [INSPIRE].

[42] R. Britto and E. Mirabella, Single cut integration, JHEP 01 (2011) 135 [arXiv:1011.2344] [INSPIRE].

[43] R. Britto and E. Mirabella, External leg corrections in the unitarity method, JHEP 01 (2012) 045 [arXiv: 1109.5106] [INSPIRE].

[44] G. Ossola, C.G. Papadopoulos and R. Pittau, Reducing full one-loop amplitudes to scalar integrals at the integrand level, Nucl. Phys. B 763 (2007) 147 [hep-ph/0609007] [INSPIRE].

[45] Z. Bern, L.J. Dixon, D.C. Dunbar and D.A. Kosower, One loop $n$ point gauge theory amplitudes, unitarity and collinear limits, Nucl. Phys. B 425 (1994) 217 [hep-ph/9403226] [INSPIRE]. 
[46] Z. Bern, L.J. Dixon, D.C. Dunbar and D.A. Kosower, Fusing gauge theory tree amplitudes into loop amplitudes, Nucl. Phys. B 435 (1995) 59 [hep-ph/9409265] [INSPIRE].

[47] R. Britto, F. Cachazo and B. Feng, Generalized unitarity and one-loop amplitudes in $N=4$ super-Yang-Mills, Nucl. Phys. B 725 (2005) 275 [hep-th/0412103] [INSPIRE].

[48] D. Forde, Direct extraction of one-loop integral coefficients, Phys. Rev. D 75 (2007) 125019 [arXiv: 0704.1835] [INSPIRE].

[49] H. Elvang and Y.-t. Huang, Scattering Amplitudes, arXiv:1308.1697 [INSPIRE].

[50] S. Caron-Huot, Loops and trees, JHEP 05 (2011) 080 [arXiv: 1007.3224] [INSPIRE].

[51] N. Arkani-Hamed, J.L. Bourjaily, F. Cachazo, S. Caron-Huot and J. Trnka, The all-loop integrand for scattering amplitudes in planar $N=4$ SYM, JHEP 01 (2011) 041 [arXiv: 1008.2958] [INSPIRE].

[52] R.K. Ellis, W.T. Giele, Z. Kunszt and K. Melnikov, Masses, fermions and generalized D-dimensional unitarity, Nucl. Phys. B 822 (2009) 270 [arXiv:0806.3467] [InSPIRE].

[53] R.K. Ellis, Z. Kunszt, K. Melnikov and G. Zanderighi, One-loop calculations in quantum field theory: from Feynman diagrams to unitarity cuts, Phys. Rept. 518 (2012) 141 [arXiv: 1105.4319] [INSPIRE].

[54] R. Pius and A. Sen, Cutkosky rules for superstring field theory, JHEP 10 (2016) 024 [arXiv: 1604.01783] [INSPIRE].

[55] A. Sen, Unitarity of superstring field theory, JHEP 12 (2016) 115 [arXiv: 1607.08244] [INSPIRE].

[56] L. Mason and D. Skinner, Ambitwistor strings and the scattering equations, JHEP 07 (2014) 048 [arXiv: 1311.2564] [INSPIRE].

[57] T. Adamo, E. Casali and D. Skinner, Ambitwistor strings and the scattering equations at one loop, JHEP 04 (2014) 104 [arXiv: 1312.3828] [INSPIRE].

[58] K. Ohmori, Worldsheet geometries of ambitwistor string, JHEP 06 (2015) 075 [arXiv: 1504.02675] [INSPIRE].

[59] Y. Geyer, L. Mason, R. Monteiro and P. Tourkine, Loop integrands for scattering amplitudes from the Riemann sphere, Phys. Rev. Lett. 115 (2015) 121603 [arXiv:1507.00321] [INSPIRE].

[60] Y. Geyer, L. Mason, R. Monteiro and P. Tourkine, One-loop amplitudes on the Riemann sphere, JHEP 03 (2016) 114 [arXiv:1511.06315] [INSPIRE].

[61] Y. Geyer, L. Mason, R. Monteiro and P. Tourkine, Two-loop scattering amplitudes from the Riemann sphere, Phys. Rev. D 94 (2016) 125029 [arXiv:1607.08887] [InSPIRE].

[62] E. D'Hoker and D.H. Phong, The geometry of string perturbation theory, Rev. Mod. Phys. 60 (1988) 917 [INSPIRE].

[63] E. Plahte, Symmetry properties of dual tree-graph n-point amplitudes, Nuovo Cim. A 66 (1970) 713 [INSPIRE].

[64] N.E.J. Bjerrum-Bohr, J.F. Donoghue and P. Vanhove, On-shell techniques and universal results in quantum gravity, JHEP 02 (2014) 111 [arXiv:1309.0804] [INSPIRE].

[65] B. Feng, R. Huang and Y. Jia, Gauge amplitude identities by on-shell recursion relation in S-matrix program, Phys. Lett. B 695 (2011) 350 [arXiv:1004.3417] [INSPIRE]. 
[66] H. Kawai, D.C. Lewellen and S.H.H. Tye, A relation between tree amplitudes of closed and open strings, Nucl. Phys. B 269 (1986) 1 [INSPIRE].

[67] Z. Bern, L.J. Dixon, M. Perelstein and J.S. Rozowsky, Multileg one loop gravity amplitudes from gauge theory, Nucl. Phys. B 546 (1999) 423 [hep-th/9811140] [INSPIRE].

[68] N.E.J. Bjerrum-Bohr, P.H. Damgaard, B. Feng and T. Sondergaard, Gravity and Yang-Mills amplitude relations, Phys. Rev. D 82 (2010) 107702 [arXiv: 1005.4367] [INSPIRE].

[69] J. Broedel, O. Schlotterer and S. Stieberger, Polylogarithms, multiple zeta values and superstring amplitudes, Fortsch. Phys. 61 (2013) 812 [arXiv:1304.7267] [INSPIRE].

[70] C.R. Mafra, Berends-Giele recursion for double-color-ordered amplitudes, JHEP 07 (2016) 080 [arXiv: 1603.09731] [INSPIRE].

[71] C. Baadsgaard et al., New representations of the perturbative S-matrix, Phys. Rev. Lett. 116 (2016) 061601 [arXiv: 1509.02169] [INSPIRE].

[72] S. He and E.Y. Yuan, One-loop scattering equations and amplitudes from forward limit, Phys. Rev. D 92 (2015) 105004 [arXiv: 1508.06027] [INSPIRE].

[73] F. Cachazo, S. He and E.Y. Yuan, One-loop corrections from higher dimensional tree amplitudes, JHEP 08 (2016) 008 [arXiv: 1512.05001] [INSPIRE].

[74] Z. Bern and D.A. Kosower, Color decomposition of one loop amplitudes in gauge theories, Nucl. Phys. B 362 (1991) 389 [inSPIRE].

[75] V. Del Duca, L.J. Dixon and F. Maltoni, New color decompositions for gauge amplitudes at tree and loop level, Nucl. Phys. B 571 (2000) 51 [hep-ph/9910563] [INSPIRE].

[76] D. Chester, Bern-Carrasco-Johansson relations for one-loop QCD integral coefficients, Phys. Rev. D 93 (2016) 065047 [arXiv:1601.00235] [InSPIRE].

[77] A. Primo and W.J. Torres Bobadilla, BCJ identities and d-dimensional generalized unitarity, JHEP 04 (2016) 125 [arXiv:1602.03161] [INSPIRE].

[78] Y.-J. Du and H. Lüo, On general BCJ relation at one-loop level in Yang-Mills theory, JHEP 01 (2013) 129 [arXiv: 1207.4549] [INSPIRE].

[79] F V. Tkachov, A theorem on analytical calculability of four loop renormalization group functions, Phys. Lett. B 100 (1981) 65.

[80] K.G. Chetyrkin and F.V. Tkachov, Integration by parts: the algorithm to calculate $\beta$-functions in 4 loops, Nucl. Phys. B 192 (1981) 159 [INSPIRE].

[81] S. Laporta and E. Remiddi, The analytical value of the electron $(g-2)$ at order $\alpha^{3}$ in $Q E D$, Phys. Lett. B 379 (1996) 283 [hep-ph/9602417] [INSPIRE].

[82] S. Laporta, High precision calculation of multiloop Feynman integrals by difference equations, Int. J. Mod. Phys. A 15 (2000) 5087 [hep-ph/0102033] [InSPIRE].

[83] Z. Bern and D.A. Kosower, Efficient calculation of one loop QCD amplitudes, Phys. Rev. Lett. 66 (1991) 1669 [INSPIRE].

[84] Z. Bern and D.A. Kosower, The computation of loop amplitudes in gauge theories, Nucl. Phys. B 379 (1992) 451 [INSPIRE].

[85] Z. Bern, L.J. Dixon and D.A. Kosower, One loop corrections to five gluon amplitudes, Phys. Rev. Lett. 70 (1993) 2677 [hep-ph/9302280] [INSPIRE]. 
[86] C. Schubert, Perturbative quantum field theory in the string inspired formalism, Phys. Rept. 355 (2001) 73 [hep-th/0101036] [INSPIRE].

[87] J. Bjornsson, Multi-loop amplitudes in maximally supersymmetric pure spinor field theory, JHEP 01 (2011) 002 [arXiv: 1009.5906] [INSPIRE].

[88] S. Badger, G. Mogull, A. Ochirov and D. O'Connell, A complete two-loop, five-gluon helicity amplitude in Yang-Mills theory, JHEP 10 (2015) 064 [arXiv:1507.08797] [INSPIRE].

[89] Z. Bern, M. Enciso, J. Parra-Martinez and M. Zeng, Manifesting enhanced cancellations in supergravity: integrands versus integrals, JHEP 05 (2017) 137 [arXiv:1703.08927] [INSPIRE].

[90] S. Mizera, Combinatorics and topology of Kawai-Lewellen-Tye relations, JHEP 08 (2017) 097 [arXiv: 1706 . 08527] [INSPIRE].

[91] E. Casali and P. Tourkine, Infrared behaviour of the one-loop scattering equations and supergravity integrands, JHEP 04 (2015) 013 [arXiv:1412.3787] [INSPIRE].

[92] M.B. Green, J.H. Schwarz and E. Witten, Superstring theory. Volume 2: loop amplitudes, anomalies and phenomenology, Cambridge University Press, Cambridge U.K. (1988).

[93] E. Panzer, Algorithms for the symbolic integration of hyperlogarithms with applications to Feynman integrals, Comput. Phys. Commun. 188 (2015) 148 [arXiv:1403.3385] [INSPIRE]. 